\title{
Development
}

\section{The Role of Even-Skipped in Drosophila Larval Somatosensory Circuit Assembly}

\author{
Zarion D. Marshall,, ${ }^{1,2}$ and ${ }^{-}$Ellie S. Heckscher ${ }^{1,2}$
}

https://doi.org/10.1523/ENEURO.0403-21.2021

${ }^{1}$ Department of Molecular Genetics and Cell Biology, University of Chicago, Chicago, IL 60637 and ${ }^{2}$ Institute for Neuroscience, University of Chicago, Chicago, IL 60637

\begin{abstract}
Proper somatosensory circuit assembly is critical for processing somatosensory stimuli and for responding accordingly. In comparison to other sensory circuits (e.g., olfactory and visual), somatosensory circuits have unique anatomy and function. However, understanding of somatosensory circuit development lags far behind that of other sensory systems. For example, there are few identified transcription factors required for integration of interneurons into functional somatosensory circuits. Here, as a model, we examine one type of somatosensory interneuron, Even-skipped (Eve) expressing laterally placed interneurons (ELs) of the Drosophila larval nerve cord. Eve is a highly conserved, homeodomain transcription factor known to play a role in cell fate specification and neuronal axon guidance. Because marker genes are often functionally important in the cell types they define, we deleted eve expression specifically from EL interneurons. On the cell biological level, using single neuron labeling, we find eve plays several previously undescribed roles in refinement of neuron morphogenesis. Eve suppresses aberrant neurite branching, promotes axon elongation, and regulates dorsal-ventral dendrite position. On the circuit level, using optogenetics, calcium imaging, and behavioral analysis, we find eve expression is required in EL interneurons for the normal encoding of somatosensory stimuli and for normal mapping of outputs to behavior. We conclude that the eve gene product coordinately regulates multiple aspects of EL interneuron morphogenesis and is critically required to properly integrate EL interneurons into somatosensory circuits. Our data shed light on the genetic regulation of somatosensory circuit assembly.
\end{abstract}

Key words: embryo; larva; mechanosensation; proprioception; somatosensation

\section{Significance Statement}

In general, even-skipped (eve) genes are considered neural cell fate determinants. Here, we show that eve gene expression is required for refinement of axon and dendrite morphogenesis and for proper functional integration of neurons into somatosensory circuits. Thus, eve coordinately regulates multiple terminal neuronal features of a class of Eve-expressing interneurons, raising the possibility that, in other neuronal contexts, eve genes regulate a similar suite of features. Our study pushes the understanding of eve beyond the level of neuron morphology to the levels of circuit physiology and whole animal behavior. It thereby provides an updated understanding of eve in development. Further, our data identify eve as a genetic entry point for future study of sensorimotor circuit assembly in Drosophila.

\section{Introduction}

Proper assembly of somatosensory circuits is critical for perception and movement (Zeilig et al., 2012). During somatosensory circuit development, interneurons wire up

Received September 28, 2021; accepted December 19, 2021; First published January 14, 2022.

The authors declare no competing financial interests. in precise patterns both with sensory neurons and with other CNS neurons (Kohsaka et al., 2017; Clark et al., 2018; D’Elia and Dasen, 2018). This is a multistep process involving, first, cell fate specification; then, axon outgrowth and dendrite

Author contributions: E.S.H. designed research; Z.D.M. and E.S.H. performed research; Z.D.M. and E.S.H. analyzed data; Z.D.M. and E.S.H. wrote the paper. 
morphogenesis; and finally, functional integration of neuronal inputs and outputs into the circuit. However, in comparison to other sensory systems (e.g., visual and olfactory), the assembly of the somatosensory system is still poorly understood (Meng and Heckscher, 2021).

The Drosophila larval nerve cord is an excellent system to study somatosensory circuit development. The organization of Drosophila and vertebrate somatosensory circuits is similar. For example, in both, a diversity of sensory neurons project axons to distinct dorsal-ventral regions (or laminae) in the CNS (Rexed, 1952; Zlatic et al., 2009). Genetically-defined subtypes of somatosensory interneurons synapse with specific sensory neurons, and those interneurons contribute to either local reflex circuits or send information to the brain (Heckscher et al., 2015; Lai et al., 2016; Wreden et al., 2017). Therefore, principles uncovered in studies of Drosophila have the potential to be broadly relevant to vertebrate somatosensory circuit development.

In Drosophila, several aspects of somatosensory circuit development are well understood. In the peripheral nervous system (PNS), specific transcription factors that regulate sensory neuron dendrite morphogenesis have been identified (Zlatic et al., 2003; Parrish et al., 2006; Hattori et al., 2007). In the CNS, early cell fate specification and the transcriptional regulation of axon guidance have been characterized (Santiago and Bashaw, 2014; Stratmann et al., 2019). However, most studies of neurons in the Drosophila CNS have focused on experimentally accessible motor neurons. Motor neurons are fundamentally distinct from somatosensory interneurons. Axons of motor neurons exit the CNS, and in general, dendrites of motor neurons do not get direct synaptic input from sensory neurons (Couton et al., 2015). In Drosophila, there remain large gaps in our understanding of the genetic control of somatosensory interneuron morphogenesis, specifically in control of dendrite morphology and circuit integration. Furthermore, it is unclear to what extent multiple terminal features, such as axon and dendrite morphology, are coordinately controlled by single transcription factors (Kurmangaliyev et al., 2019).

In this study, as a model, we focus on Drosophila larval laterally placed interneurons (ELs). ELs are named for their expression of the transcription factor, Even-skipped (Eve) and their lateral cell body position. EL interneurons all process somatosensory stimuli and are necessary for normal behavior (Heckscher et al., 2015). We focus on

This work was supported by the National Institutes of Health Grant R01NS105748 and the University of Chicago, Department of Molecular Genetics and Cell Biology Start-up funds (to E.S.H.).

Acknowledgements: We thank Chip Ferguson and Paschalis Kratsios (University of Chicago), Chris Doe (University of Oregon), and members of the Heckscher lab for comments that improved this manuscript. We also thank Richard Baines (Manchester) for sharing Eve binding site data.

Correspondence should be addressed to Ellie S. Heckscher at heckscher@ uchicago.edu.

https://doi.org/10.1523/ENEURO.0403-21.2021

Copyright (C) 2022 Marshall and Heckscher

This is an open-access article distributed under the terms of the Creative Commons Attribution 4.0 International license, which permits unrestricted use, distribution and reproduction in any medium provided that the original work is properly attributed.
ELs because in comparison to other Drosophila somatosensory interneurons, the developmental origins of ELs are known, and reagents to label ELs in embryos and larvae are available (Fujioka et al., 1999). To study the transcriptional control of EL development, we took a candidate gene approach focusing on the role of eve. eve encodes a conserved homeobox transcription factor. eve or its homologs are expressed in neurons in animals across phyla (Ferrier et al., 2001; Heckscher et al., 2015). We reasoned that eve is likely to play an important role in ELs because cell type-specific transcription factor genes often are important in the cell types they define. Further, in general, eve and its homologs play roles in cell fate specification and axon guidance (Doe et al., 1988; Landgraf et al., 1999; Moran-Rivard et al., 2001; Esmaeili et al., 2002; Fujioka et al., 2003; Pym et al., 2006; Zarin et al., 2014; Juárez-Morales et al., 2016). Here, we show eve gene expression is required for multiple aspects of terminal neuronal development and for proper functional integration of ELs into sensorimotor circuits. Specifically, eve regulates neurite branching, axon extension, dendrite positioning, formation of functional inputs, and mapping of functional outputs, as well as whole animal behavior. Thus, we uncover several, previously undescribed roles for eve in neuronal physiology, and we identify eve as a transcriptional regulator of Drosophila somatosensory circuit assembly.

\section{Materials and Methods}

\section{Fly genetics}

Standard methods were used to propagate fly stocks. Unless otherwise noted, larvae were raised at $25^{\circ} \mathrm{C}$ and fed yeast paste containing water and yeast $(5: 3$ ratio by weight). For optogenetics experiments, yeast paste containing 100- $\mu$ l all trans retinal (ATR) was used. For a list of stocks used in this study see Table 1.

\section{Species sex}

In these experiments, embryos and early-stage larvae were used. At these developmental stages, flies have no distinguishing sexual characteristics. Thus, all experiments were conducted in a manner that was blind to sex.

\section{Immunostaining}

We used standard methods (Meng et al., 2019, 2020). Larval brains were pulled at ambient temperature within a 7 -min window. Brains were fixed in freshly prepared $1 \times$ phosphate buffered saline containing 4\% formaldehyde for 7-10 min. Many primary antibodies were obtained from Developmental Studies Hybridoma Bank, created by the NICHD of the NIH and maintained at The University of lowa, Department of Biology. See Table 1 for primary antibodies. Secondary antibodies were from Jackson ImmunoResearch and were used according to manufacturer's instructions. Images were acquired on a Zeiss 800 confocal microscope with $40 \times$ objective. Embryos were staged for imaging based on standard morphologic criteria. 
Table 1: Methods used to label EL interneurons

\begin{tabular}{|c|c|c|c|c|c|}
\hline \multirow[b]{2}{*}{ Genotype } & & \multicolumn{2}{|l|}{ Labeling method } & \multirow[b]{2}{*}{$\begin{array}{l}\text { UAS-FLP, actin-FRT-stop- } \\
\text { FRT-GAL4;;EL-GAL4 }\end{array}$} & \multirow[b]{2}{*}{ 11F02-GAL4 } \\
\hline & & Anti-Eve & $E L-G A L 4$ & & \\
\hline \multirow[t]{2}{*}{ Wild type } & Neurons labeled & $\begin{array}{r}\text { EL interneurons, } \\
\text { Motor neurons }\end{array}$ & EL interneurons & EL interneurons & $\begin{array}{l}\text { Late-born ELs, other } \\
\text { neurons }\end{array}$ \\
\hline & Stages & $\begin{array}{l}\text { Neuron birth to } \\
\text { larval L3 }\end{array}$ & $\begin{array}{l}\text { Embryos stage } 14 \text { to } \\
\text { larval L2 }\end{array}$ & $\begin{array}{l}\text { Embryo stage } 15 \text { to } \\
\text { larval L3 }\end{array}$ & $\begin{array}{l}\text { Embryo stage } 15 \text { to } \\
\text { larva }\end{array}$ \\
\hline \multirow[t]{2}{*}{$\begin{array}{l}\text { EL eve mutant/ } \\
\text { EL eve mutant }\end{array}$} & Neurons labeled & Motor neurons & EL interneurons & EL interneurons & $\begin{array}{l}\text { Late-born ELs, other } \\
\text { neurons }\end{array}$ \\
\hline & Stages & $\begin{array}{l}\text { Neuron birth to } \\
\text { larval L3 }\end{array}$ & $\begin{array}{l}\text { Embryo stage } 14 \text { to } \\
\text { stage } 15\end{array}$ & $\begin{array}{l}\text { Embryo stage } 15 \text { to } \\
\text { larva L3 }\end{array}$ & $\begin{array}{l}\text { Embryo stage } 15 \text { to } \\
\text { larva }\end{array}$ \\
\hline \multirow[t]{2}{*}{$\begin{array}{l}\text { EL eve mutant/ } \\
\text { eve(5) }\end{array}$} & Neurons labeled & $\begin{array}{l}\text { EL interneurons, } \\
\text { Motor neurons }\end{array}$ & EL interneurons & $\mathrm{N} / \mathrm{A}$ & $\mathrm{N} / \mathrm{A}$ \\
\hline & Stages & $\begin{array}{l}\text { Neuron birth to } \\
\text { larval L3 }\end{array}$ & $\begin{array}{l}\text { Embryo stage } 14 \text { to } \\
\text { larval L2 }\end{array}$ & $\mathrm{N} / \mathrm{A}$ & N/A \\
\hline
\end{tabular}

\section{Four ways to label ELs interneurons}

In this study, we use four ways to label ELs (Table 2).

\section{Eve antibody staining}

In wild-type, anti-Eve labels motor neurons and EL interneurons. In EL eve mutants, anti-Eve staining is lost from the ELs. Notably, in EL eve mutants in trans to a hypomorphic eve allele, eve(5), which generates a truncated Eve protein, anti-Eve can be used to track ELs.

\section{EL-GAL4}

In wild-type, EL-GAL4 expresses in ELs from the middle of embryogenesis until the middle of larval development. However, in EL eve mutants, EL-GAL4 drives only a pulse of gene expression. Thus, in ELs, Eve is dispensable for initiation of EL-GAL4 expression, but is required to maintain EL-GAL4 expression.

\section{EL-GAL4 with a permanent labeling cassette}

To positively mark ELs in an EL eve mutant background using EL-GAL4, we added a FLP-based permanent labeling cassette (UAS-FLP, actin-FRT-Stop-FRT-GAL4).

\section{F02-GAL4}

In wild-type, 11F02-GAL4 drives expression in the lateborn subset of ELs, as well as a few other uncharacterized neurons (Heckscher et al., 2014). 11F02-GAL4 expression is unchanged in EL eve mutants.

\section{Larval behavior}

$\mathrm{L} 1$ larvae were collected from 0 to $6 \mathrm{~h}$ after hatching. All larvae were rinsed in mesh chambers under dH2O until they were freed from food debris. Collected larvae were placed on $5-\mathrm{mm}$-thick $2 \%$ agarose gels set in $5.5-\mathrm{cm}-$ wide Petri dishes at least $15 \mathrm{~min}$ before recording. All recordings were done at $22-25^{\circ} \mathrm{C}$. Five to 30 larvae were allowed to freely crawl on a gel per recording. For pinching assay, pinches were delivered to the side of the larval body wall with a pair of forceps. A larva was considered to be rolling if the trachea disappeared under one side of the larva body and reappeared on the other side. Note that this criterion for a roll is not based on larval speed. For hunching assay, a vibration was delivered to the larvae using the speaker and sound described below. Hunches were considered to be a shortening of the distance between the head and body center associated with a pause in crawling, but not a head turn. For left-right asymmetry and crawling speed assays, larvae were recorded in our custom-built behavior rig (Wreden et al., 2017). Images were acquired at 10 frames per second. For optogenetic experiments, all larvae were recorded 48-54 h after hatching. A total of 10-100 larvae were placed on a rectangular 5 -mm-thick agarose gel at least $15 \mathrm{~min}$ before recording. Recordings began with a 30-s period of no stimulus followed by a 30 -s period of stimulating light and ending with a final 30-s period of no stimulus. For further setup details, please refer to Wreden et al. (2017).

Larvae were tracked using FIMTracker (Risse et al., 2017) software using default parameters including five spline points, this includes centroid, head, and tail points, plus two other points, one halfway between head and centroid and one halfway between the tail and centroid. Larvae were discarded from analysis if (1) the larva's track was $<300$ frames; (2) the larva was improperly masked; or (3) if occasionally the larva collided with another larva and it was not rectified by thresholding. Tracks were then analyzed using custom scripts (Heckscher et al., 2015) on MATLAB (MathWorks). Speeds were calculated using the center spline point over a 10 frame (1-s) window. Statistical tests were performed using Prism 9 software (GraphPad).

\section{Calcium imaging}

For calcium imaging experiments, all larvae were within $6 \mathrm{~h}$ of age on the day of recording and collected 48-54 $\mathrm{h}$ after hatching. Larvae expressing GCaMP6m (UAS-FLP, act5C-FRT.stop-GAL4; $\triangle E L, D f(2 R) e v e /+$; EL-GAL4/UASGCaMP6m or UAS-FLP, act5C-FRT.stopGAL4; $\triangle E L, D f(2 R)$ eve/ $\Delta E L, D f(2 R)$ eve; EL-GAL4/UASGCaMP6m) were rinsed with water and placed ventral side up on agarose pads with a $22 \times 22 \mathrm{~mm}$ coverslip placed on top. Pads were made by pouring $3 \%$ agarose into a well. Recordings began with a $30-\mathrm{s}$ period of no stimulus followed by a 30-s period of sound stimulus and ending with a final 30-s period of no stimulus. A Visaton FR12, 4 Ohm speaker (5 inches in diameter) and a PYLE PCA2 stereo power amplifier was used to project sound. 
Table 2: Antibodies, fly lines

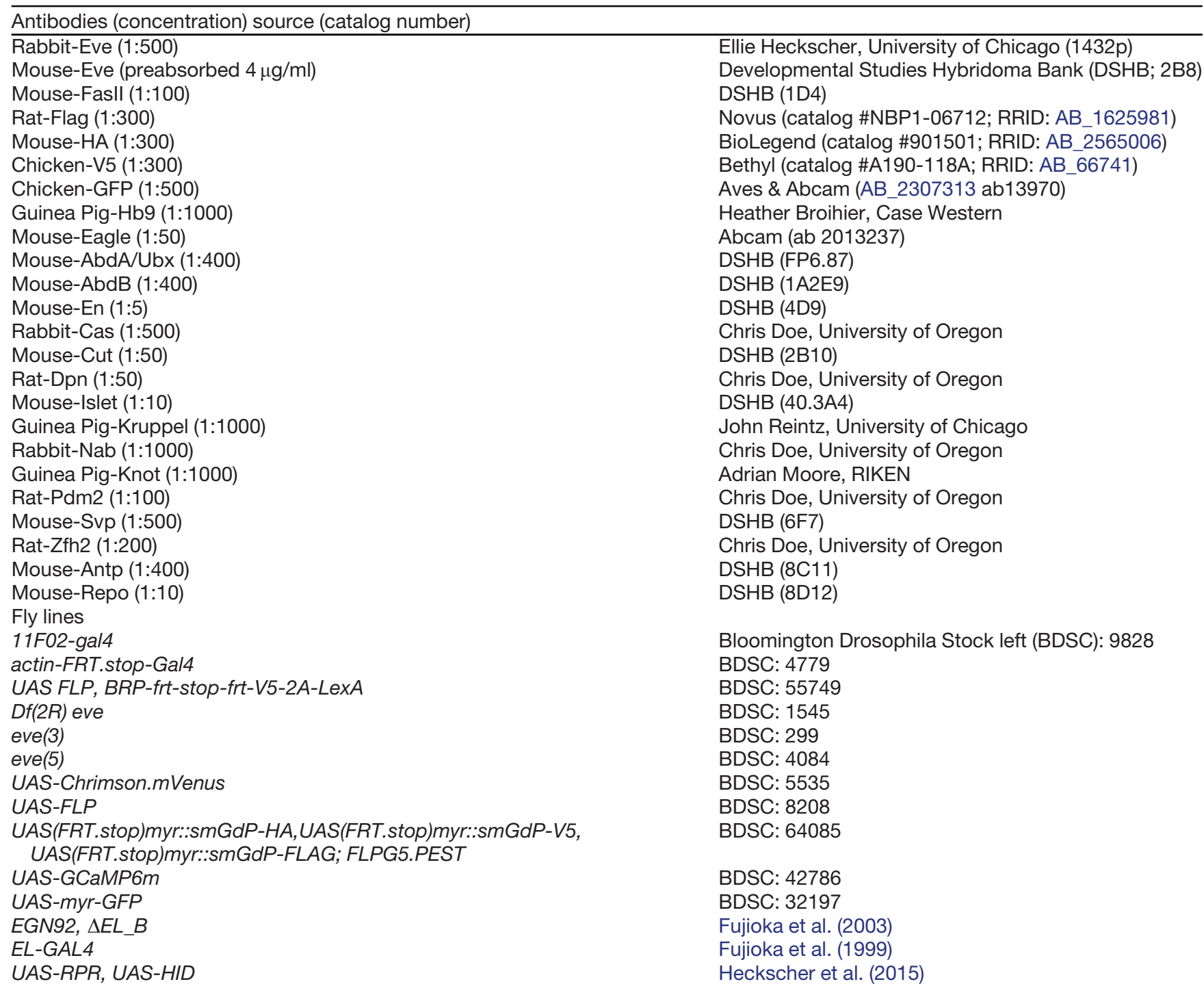

For further details, refer to Wreden et al., 2017. Images were acquired on a Zeiss LSM 800 confocal microscope using $0.1-0.2 \%$ 488-nm laser power with the pinhole entirely open. Images were acquired at three frames per second using a $10 \times(0.3 \mathrm{NA})$ or $20 \times(0.8 \mathrm{NA})$ objective. The calcium signal was continuously collected before, during, and after the stimulus. Extracting changes in GCaMP6m fluorescence amplitude was done using Fiji as in Wreden et al. (2017). A region of interest (ROI) that included the larval nerve cord was manually drawn, and the mean fluorescence within the ROI was calculated for each time point.

\section{Single neuron labeling}

We labeled single neurons using MultiColor FLP Out (MCFO; Nern et al., 2015). MCFO stochastically labels the membranes with epitopes in cells within a GAL4 pattern. MCFO uses FLP recombinase, which we also use to label larval EL neurons in EL eve mutants (Table 2). So, we cannot use these reagents together. Here, for MCFO, we used 11F02-GAL4, which is expressed in late-born ELs and three poorly characterized non-EL interneurons directly adjacent to ELs (Heckscher et al., 2014; Wreden et al., 2017). For 11F02-GAL4 to be useful, we needed to distinguish between ELs and non-ELs in wild-type and EL eve mutant backgrounds. In both backgrounds, we performed single neuron MCFO labeling. In both backgrounds, we can identify three non-EL neurons based on morphology, which we call: 11F02d, 11F02m, and 11F02z. We conclude that loss of Eve from ELs does not have gross nonautonomous effects on non-EL neurons. We also conclude that we can distinguish between EL and non-EL neurons in EL eve mutants. To obtain single cell clones, adult flies were allowed to lay eggs for 24 h on apple juice caps. Caps were heat shocked in a water bath at $37-39^{\circ} \mathrm{C}$ for $15-30 \mathrm{~min}$ and incubated at $25^{\circ} \mathrm{C}$ for 4-5 h. First instar larvae were dissected. Their brains were stained for HA, Flag, and V5 epitopes to visualize single cell clones. Larvae were also stained for Eve protein to confirm the identity of each single cell clone as an EL, and 


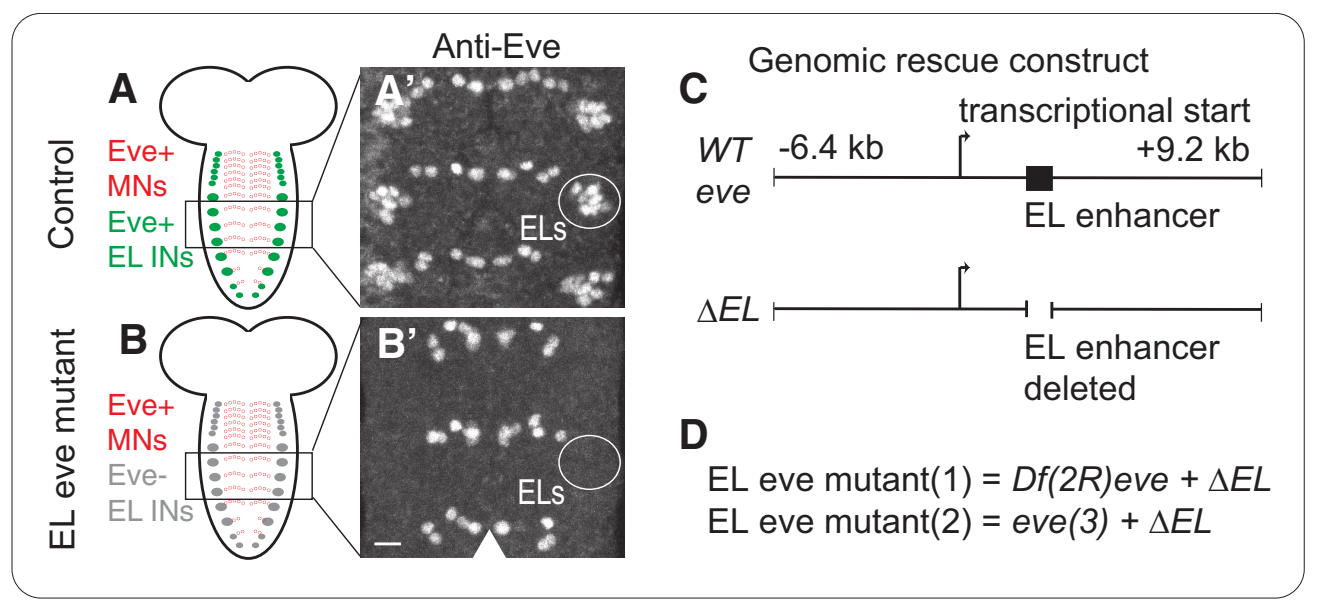

Figure 1. In EL eve mutants, EL interneurons lack Eve expression. $\boldsymbol{A}, \boldsymbol{B}$, Images of Eve expression in the nerve cord of Drosophila embryos. $\boldsymbol{A}$, In control, each segment has Eve(+) motor neurons (MNs; red) and Eve(+) EL interneurons (INs; green). B, In EL eve mutants, Eve is selectively lost from EL, but neurons themselves remain (Fujioka et al., 2003). Images show Eve expression in three segments of the Drosophila nerve cord of stage 16 embryos. Anterior is up with scale bar of $15 \mu \mathrm{m}$. Position of EL interneurons in one hemisegment is circled. Midline is marked by an arrowhead. $\boldsymbol{C}, \boldsymbol{D}$, Schematics of genomic constructs that rescue eve expression. $\boldsymbol{C}$, The top line shows a wild-type "WT eve" genomic DNA fragment (EGN92; Fujioka et al., 2003), which contains all known eve coding and regulatory sequences. The bottom line represents the " $\Delta E L$ " rescue construct, which is identical to WT eve construct except it lacks the EL enhancer (EGN92 $\Delta \mathrm{EL}$; Fujioka et al., 2003). $\boldsymbol{D}$, Two different eve null alleles, $D f(2 R)$ eve and eve(3), are rescued with the $\Delta \mathrm{EL}$ construct, referred to EL eve mutant (1) and EL eve mutant (2), respectively. Genotypes: control is $\Delta E L, D f(2 R) e v e /+$ and EL eve mutant is $\Delta E L$, eve(3)/ $\Delta E L, D f(2 R) e v e$.

to assign segmental identity to each clone. Clones were imaged on a Zeiss 880 or 800 confocal microscope. We generated $>115$ single-cell clones. Among these clones, we saw each neuronal morphology in a minimum of two independently derived clones (i.e., larvae). Each clone was analyzed in dorsal and posterior views.

\section{Results}

\section{In EL eve mutants, EL interneurons lack eve expression}

The objective of this study was to determine the role of the transcription factor Eve in EL somatosensory interneurons. eve is an essential gene in Drosophila. It is expressed in stripes in early embryos, in other developing tissues, as well as in a subset of motor neurons and EL interneurons (Frasch et al., 1987; Heckscher et al., 2014; Fig. 1A). In this study, we removed eve gene expression specifically from ELs using "EL eve mutants," which were previously generated by Fujioka and colleagues (Fujioka et al., 2003). Briefly, a genomic construct containing all eve regulatory elements was generated (Fig. 1C). This construct rescues eve null mutants to viability, and eve is expressed at normal levels and in normal locations (Fujioka et al., 2003). Fujioka and colleagues deleted from the construct a regulatory region sufficient to drive eve expression in ELs, thereby making a " $\Delta E L$ " construct (Fig. 1C; Fujioka et al., 1999, 2003). When eve null alleles are rescued with $\Delta E L$, eve is expressed at normal levels and locations everywhere except ELs interneurons, which completely lack Eve protein (Fig. 1B; Fujioka et al., 2003).

\section{eve regulates multiple aspects of interneuron morphogenesis}

eve or its homologs (e.g., evx genes) often regulate neuronal axonal pathfinding. For example, in mouse spinal cord, evx1 is required for V0v interneurons to send axons across the midline (Moran-Rivard et al., 2001). In Drosophila or Caenorhabditis elegans motor neurons, loss of eve genes results in dramatic changes in axon trajectories (Esmaeili et al., 2002; Fujioka et al., 2003). In contrast, in Zebrafish, evx1/2(-) V0v interneurons have normal axonal morphology (Juárez-Morales et al., 2016). These data demonstrate that eve plays a context dependent role in axons and raise the question to what extent eve regulates EL interneuron axon morphogenesis in Drosophila larvae.

In embryos, we characterized EL morphology by expressing a membrane localized GFP. In stage 15 embryos, in both control and EL eve mutants, EL axons cross the midline (Fig. 2A-C). In stage 16 embryos, we additionally stained with anti-Fas2 to visualize three fascicles in the neuropile-lateral, intermediate, and medial (Landgraf et al., 2003). In control, after midline crossing, most ELs grow laterally until reaching the intermediate fascicle and extend toward the anterior. In a subset of segments, ELs extend to the anterior along both intermediate and medial fascicles (Fig. 2D,F). In EL eve mutants, however, a larger proportion of ELs extend along both the intermediate and medial fascicles. Further, in some segments, ELs project along the intermediate and lateral fascicles, a phenotype never observed in controls (Fig. 2E,F). In stage 17 embryos (the final stage of embryogenesis), in control, ELs project so far to the anterior that they reach the next segment, making a ladder like pattern (Fig. 2G). In contrast, in EL eve mutants, only a small proportion of Eve(-) ELs reach the next segment (Fig. $2 \mathrm{H}, \mathrm{l})$.

At larval stages, ELs have mature morphologies and are incorporated into functioning somatosensory circuits. We labeled individual ELs, using MCFO, which allows us to determine which part of the EL, axon, dendrite, or both, is impacted by loss of eve expression (Nern et al., 2015). We 


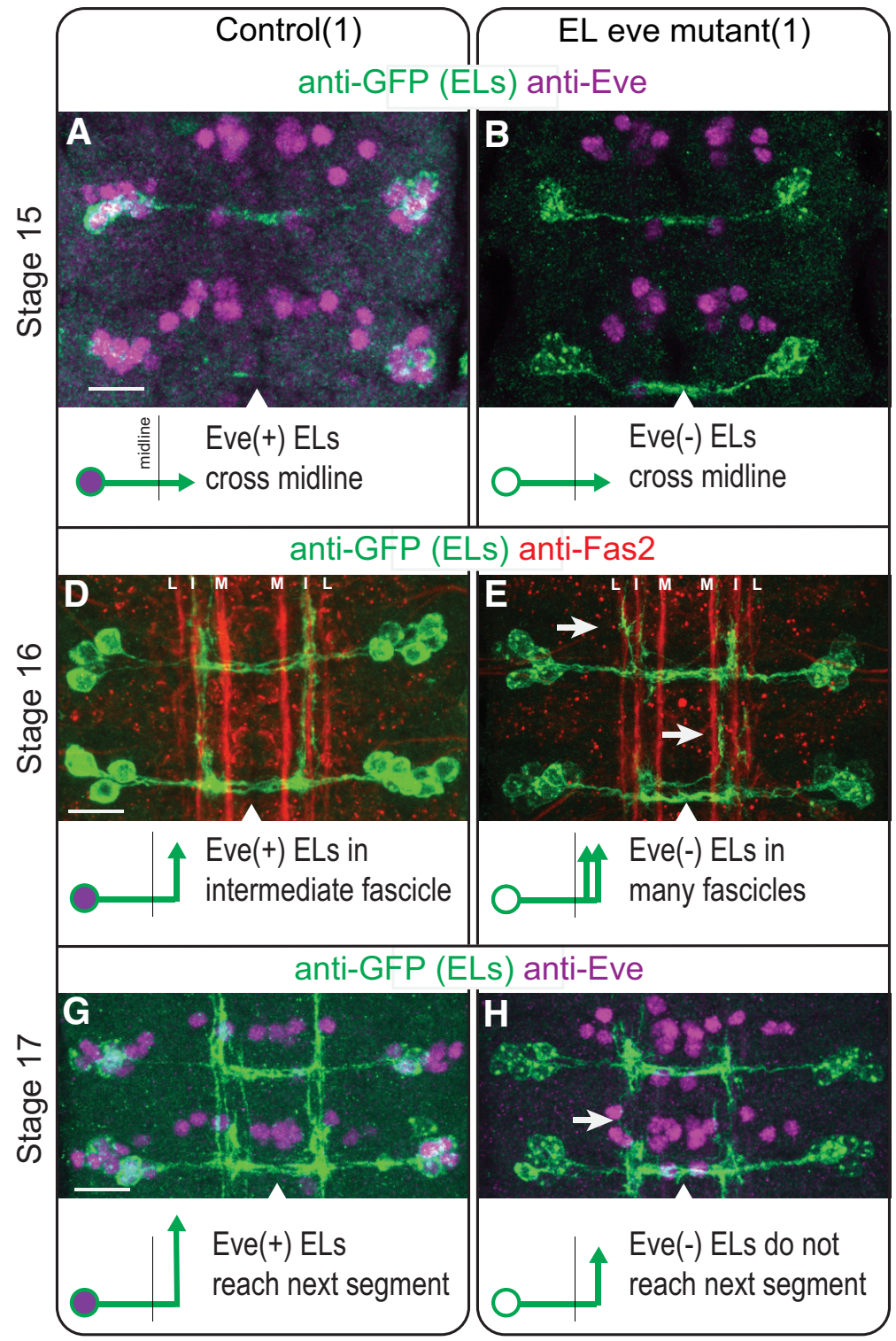
C ELs crossing midline

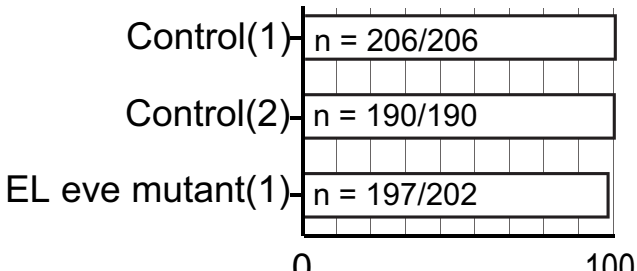
$\%$ hemisegments with processes crossing midline

F ELs in intermediate fascicle

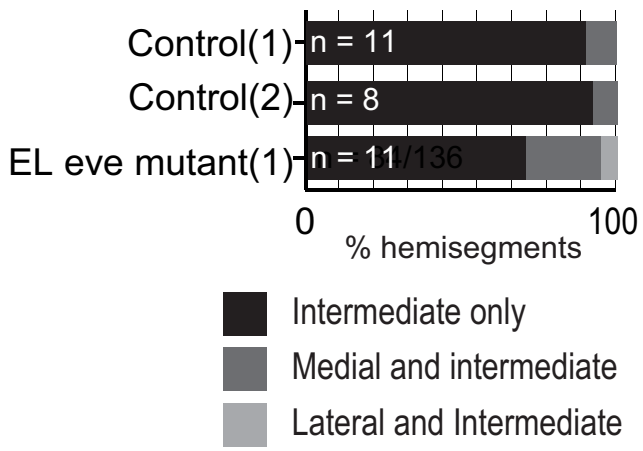

I ELs reach next segment

\begin{tabular}{|c|c|c|}
\hline Control(1) & $n=58 / 70$ & \\
\hline Control(2)- & $n=53 / 68$ & \\
\hline EL eve mutant(1) & $n=13 / 79$ & \\
\hline$\%$ hem & & \\
\hline
\end{tabular}

Figure 2. In embryos, eve expression is required for proper medial-lateral and anterior-posterior neurite positioning of EL interneurons. $\boldsymbol{A}-\boldsymbol{C}$, Images and quantification of stage 15 embryos with EL interneurons extending axons across the midline. $\boldsymbol{A}$, $\boldsymbol{B}$, In control and EL eve mutants, ELs extend across the midline. Two abdominal segments are shown with midline noted as an arrowhead. Anterior is up and scale bars are $10 \mu \mathrm{m}$. Below each image is an illustration of the phenotype. $\boldsymbol{C}$, For this quantification, $n=$ number of hemisegments with EL processes crossing the midline over the total number of hemisegments scored. $\boldsymbol{D}-\boldsymbol{F}$, Images and quantification of stage 16 embryos with defects in medial-lateral positioning of EL neurites in EL eve mutants. $\boldsymbol{D}$, In control, ELs project toward the anterior mainly along the intermediate fascicle. $\boldsymbol{E}$, In EL eve mutants, ELs project in additional fascicles (arrows; $L=$ lateral fascicle, I = intermediate, $\mathbf{M}=$ medial). $\boldsymbol{F}$, For this quantification, $n=$ number of hemisegments scored. $\mathbf{G}-\mathbf{I}$, Images and quantification of stage 17 embryos, with defects in anterior-posterior positioning of EL neurites in EL eve mutants. G, In control, ELs extend neurites to the next anterior segment. $\boldsymbol{H}$, In EL eve mutants, neurites do not extend to the next segment (arrow). I, For this quantification, $n=$ number of hemisegments with processes reaching the next segment over the total number of hemisegments scored; chi-squared test, ${ }^{* * * *} p<0.0001$. Genotypes: control 1 is EL-GAL4/UAS-myr-GFP; control 2 is UAS-FLP, act5C-FRT.stop-GAL4;;ELGAL4/UAS-myr-GFP. EL eve mutant (1) is UAS-FLP, act5C-FRT.stop-GAL4; $\triangle E L, D f(2 R) e v e / \Delta E L, D f(2 R) e v e ; ~ E L-G A L 4 / U A S-m y r-G F P$.

restricted our analysis to local, late-born ELs, because they were most abundantly labeled in our dataset (Fig. $3 A)$. First, we focused our analysis along medial-lateral and anterior-posterior axes because in Figure 2 we detected defects along these axes. We used Sholl analysis to count the number of intersections between an EL neurite and concentric circles with increasing radii $(1-\mu \mathrm{m}$ intervals) centered on the soma (Sholl, 1953). We plotted the median, minimum, and maximum number of intersections versus circle radius to generate a description of arborization. In control, local, late-born ELs have two sets of branching neurites off the main neurite. Ipsilaterally (same side) dendritic neurites are found $\sim 20 \mu \mathrm{m}$ from the soma. Contralaterally (opposite side) axonal neurites branch 

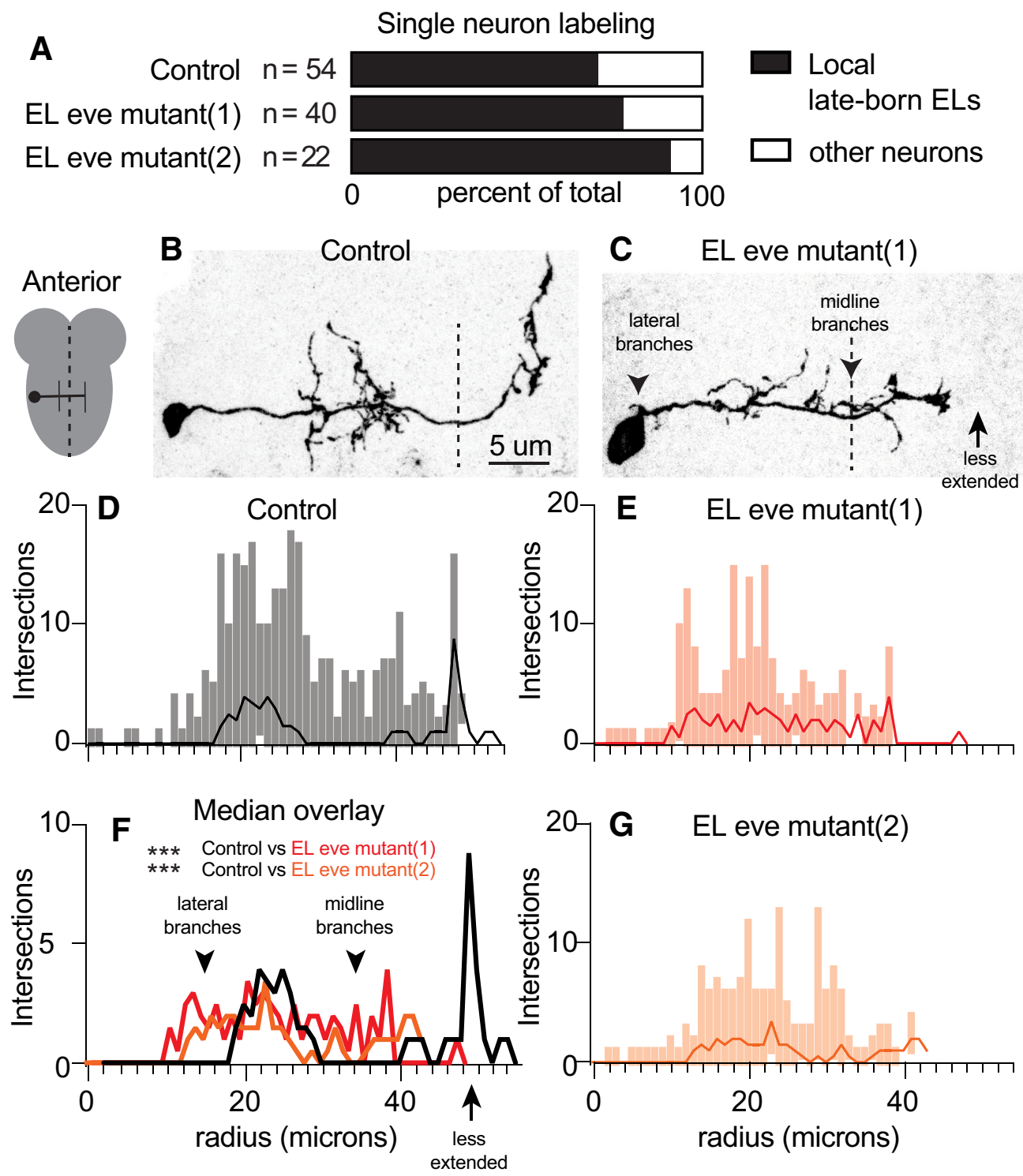

Figure 3. In larvae, Eve(-) ELs have excessive branching off the main neurite and diminished axon extension. A, Quantification of labeled neurons. In all genotypes, the most numerous type of singly-labeled neurons are local, late-born ELs. $n=$ total number of singly-labeled neurons for each genotype. B, C, Images of singly-labeled ELs. Drosophila neurons are pseudo-unipolar. B, In control, dendrites are located ipsilateral to the soma. On the contralateral side, axons turn to the anterior and form branches where output synapses are found (Heckscher et al., 2015). C, In EL eve mutants, there is excessive branching off the main neurite (arrowheads), and the axon is less extended (arrow). Anterior is up with a scale bar of $5 \mu \mathrm{m}$. Dashed line indicates midline. $\boldsymbol{D}-\mathbf{G}$, Quantifications of neuron morphology. For each plot, the $x$-axis is the radius of a concentric circle centered on the EL soma. The $y$-axis is number of times a singly-labeled EL intersects a circle. Median (dark line) and range (lighter bars) are shown. $\boldsymbol{F}$, Control is black; red and orange lines are EL eve mutants (1) and (2), respectively. Wilcoxon test, ${ }^{* *} p<0.0001$. Genotypes: control is $11 F 02-G A L 4 / U A S-$ MCFO. EL eve mutant (1) is $\triangle E L, D f(2 R)$ eve/ $\triangle E L, D f(2 R)$ eve; 11F02-GAL4/UAS-MCFO. EL eve mutant (2) is $\triangle E L, D f(2 R) e v e / \triangle E L$, eve (3); 11F02-GAL4/UAS-MCFO.

$\sim 50 \mu \mathrm{m}$ from the soma (Fig. 3B,D). In EL eve mutants, neurites of Eve(-) ELs branched excessively off the main neurite near the soma and in the midline, which is never seen in wild type (Fig. 3C, arrowheads). Furthermore, Eve (-) EL axons are less extended, branching at $\sim 40 \mu \mathrm{m}$ from the soma (Fig. 3C, arrow). These changes are reflected in a statistically significant difference in the distribution of intersections (Fig. 3F).

Next, in the same set of larval clones as shown in Figure 3, we characterized ELs along the dorsal-ventral axis. For somatosensory interneuron dendrites, positioning along the dorsal-ventral axis is particularly important because different sensory neurons axons project to different dorsal-ventral domains within the CNS (Landgraf et al., 2003). Therefore, dorsal-ventral positioning of somatosensory interneuron dendrites is expected to dictate the types of sensory input received by a given interneuron. Currently, the molecular control of dorsal-ventral dendrite positioning of somatosensory interneurons is extremely poorly understood. Here, we counted the number of 
A

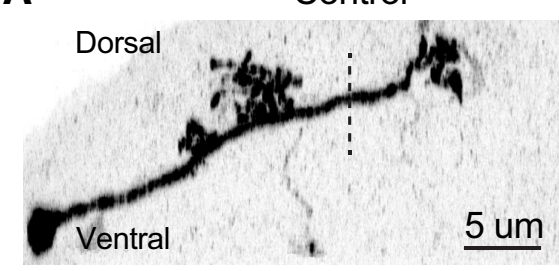

B

EL eve mutant(1)
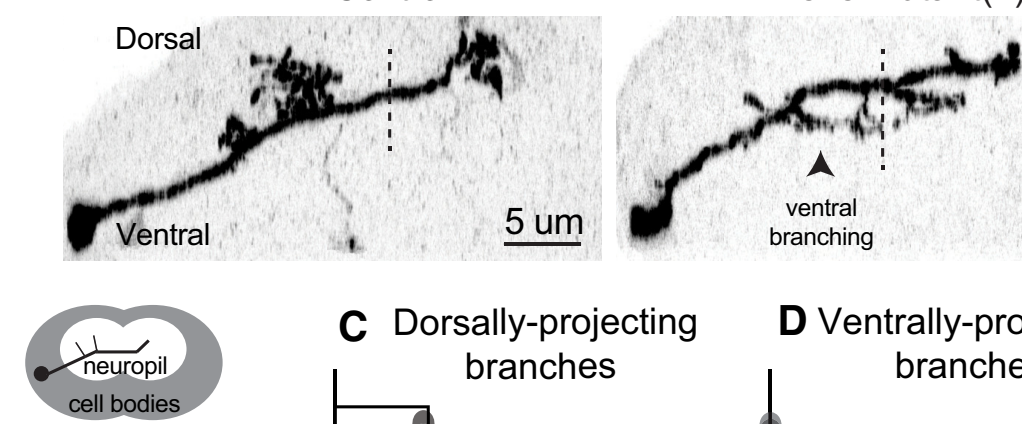

C Dorsally-projecting branches

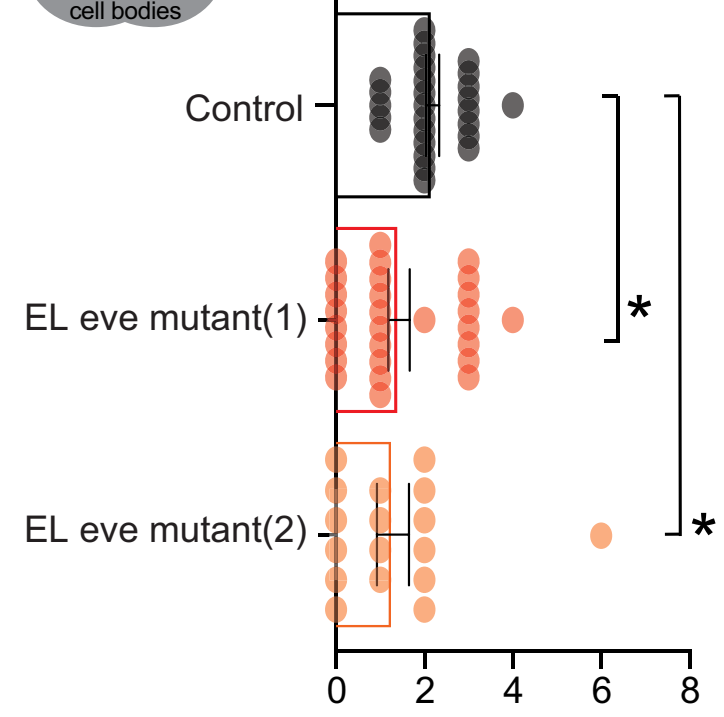

Number of branches

\begin{abstract}
D Ventrally-projecting
\end{abstract}

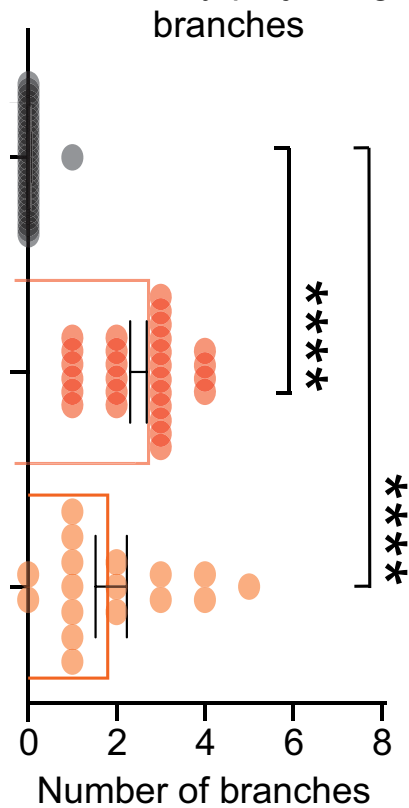

Figure 4. Eve(-) ELs have mispositioned dorsal-ventral dendrites. $\boldsymbol{A}, \boldsymbol{B}$, Images of singly-labeled ELs in side view. $\boldsymbol{A}$, In control, ELs have ipsilateral dendrites that project dorsally. $\boldsymbol{B}$, In EL eve mutants, many branches project ventrally. These are the same neurons as in Figure $3 B, C$, except here dorsal is up. $\boldsymbol{C}, \boldsymbol{D}$, Quantification of dendrite orientation. The number of branches pointing dorsally or ventrally is plotted with each dot representing a single neuron. Bars show average, and whiskers show standard deviation. ANOVA with Dunnett's multiple comparison; ${ }^{*} p<0.05$ and ${ }^{* * * *} p<0.0001$.

dorsally and ventrally projecting ipsilateral branches (i.e., dendrite). In control, nearly all Eve(+) ELs have dorsally, but not ventrally, projecting dendrites (Fig. $4 A, C, D$ ). In EL eve mutants, there is a significant reduction in dorsally projecting dendrites and a significant increase in ventrally projecting dendrites for Eve(-) ELs (Fig. 4B-D).

Finally, we asked whether loss of eve expression transforms the ELs into another interneuron type. To do so, we took both anatomic and genetic approaches. Anatomically, we mined the Drosophila larval connectome (Ohyama et al., 2015). We looked at each local neuron in the first abdominal segment, but found no neurons with morphology matching that of $\mathrm{Eve}(-) \mathrm{EL}$ interneurons. Genetically, we looked for large-scale changes in gene expression, which often accompany cell fate changes. We surveyed 19 genes that were candidates to be regulated by eve in ELs. These candidates included genes regulated by eve in non-EL cell types (En, Fasll, HB9, Islet; see Fig. 5A,B), genes expressed in ELs (Castor, Eagle, Zfh2, Knot, Kruppel, Nab, Pdm2, Seven-up; see Fig. 5C,D), and genes with putative Eve binding sites (Antp, AbdA/Ubx, AbdB, Cut, Dpn, Repo;
Broihier and Skeath, 2002; Pym et al., 2006). There are no obvious changes in expression for any of these genes in Eve(-) ELs. Taken together, these data support the conclusion that, in ELs, Eve is not repressing alternative interneuron fate.

In summary, we find that, in ELs, eve expression is not required for the initial step of EL morphogenesis-axon midline crossing. However, eve is required for later stages of EL morphogenesis, refining morphology in all three axes, medial-lateral, anterior-posterior, and dorsal-ventral. Further, eve is required in both axons and dendrites. Thus, in EL interneurons, eve expression coordinately regulates multiple aspects of morphogenesis. Finally, we find no evidence to support the idea that, in ELs, Eve represses alternative neuronal fates because Eve(-) EL interneurons do not resemble any other wild-type interneuron.

\section{Loss of eve expression disrupts EL somatosensory stimulus encoding}

In wild-type animals, EL interneurons encode somatosensory stimuli. Late-born ELs get direct synaptic input from proprioceptive sensory neurons and indirect proprioceptive 


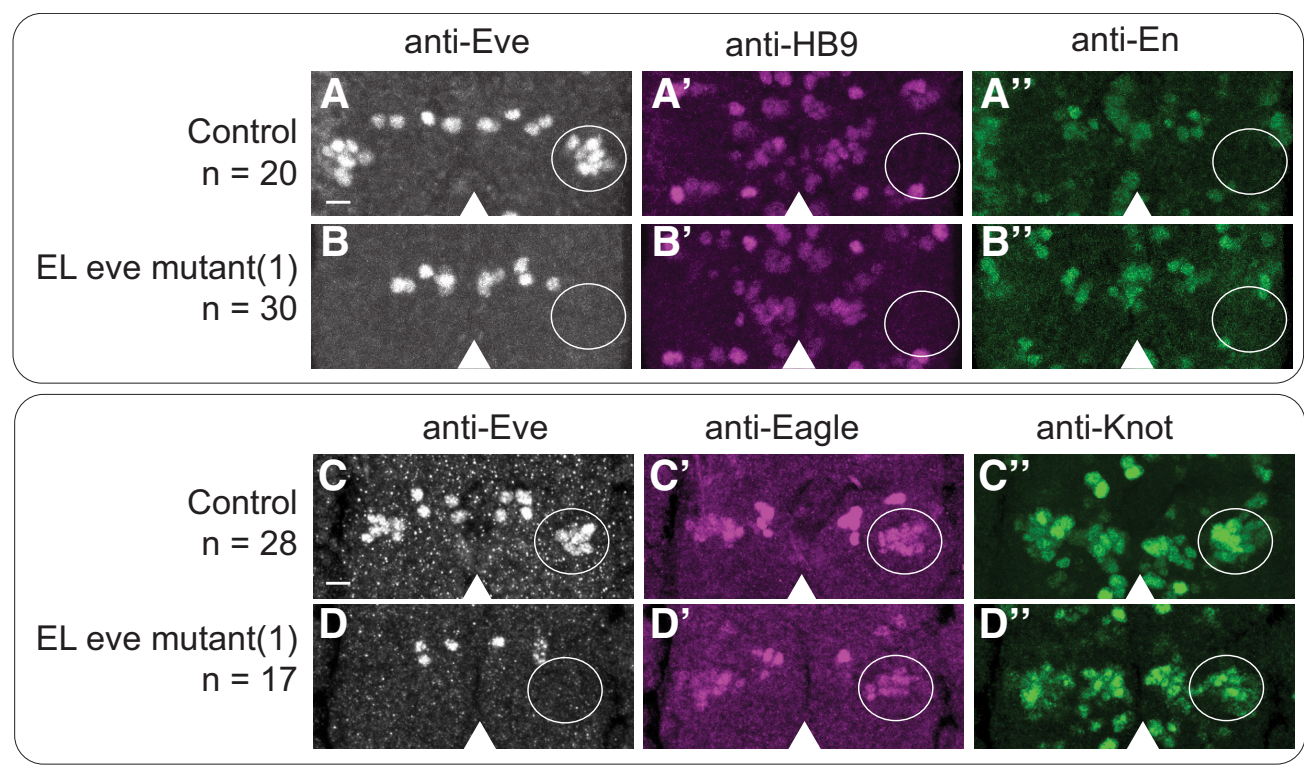

Figure 5. Eve(-) ELs do not derepress molecular markers. $\boldsymbol{A}-\boldsymbol{D}$, Images of marker gene expression in ELs. $\boldsymbol{A}, \boldsymbol{C}$, In control, ELs lack expression of ventral motor neuron maker, HB9 $\left(\boldsymbol{A}^{\prime}\right)$ and an interneuron marker En $\left(\boldsymbol{A}^{\prime \prime}\right)$. ELs express both Eagle $\left(\boldsymbol{C}^{\prime}\right)$ and Collier $\left(\boldsymbol{C}^{\prime \prime}\right)$. $\boldsymbol{B}, \boldsymbol{D}$, In EL eve mutants, there is no change in marker gene expression. Representative segments of stage 16 embryos. Anterior is up with a scale bar of $5 \mu \mathrm{m}$. Arrowhead shows midline. Area containing EL neurons is circled. $n=$ number of hemisegments scored. Each row shows separate image channels of the same co-stained stained embryo. Genotypes: control is wild-type and EL eve mu$\operatorname{tant}(1)$ is $\Delta E L, D f(2 R) e v e / \Delta E L, \operatorname{Df}(2 \mathrm{R})$ eve.

input via Jaam interneurons (Fig. 6A; Heckscher et al., 2015). Early-born ELs get direct synaptic input from vibration sensitive (chordotonal) sensory neurons and indirect chordotonal input via Basin interneurons (Fig. 6B; Wreden et al., 2017). The observation that Eve(-) EL dendrites are mispositioned raised the possibility that eve is required for ELs to properly encode somatosensory stimuli (Fig. 4). We test this idea here.

Mispositioned dendrites in Eve(-) EL interneurons likely disrupt the ability of ELs to form connections with their normal sensory neuron input partners (Fig. 5C,E). Alternatively, ELs could still form synapses with input partners via a compensatory mechanism (Fig. 6F). It is essential to consider this alternative because a recent study revealed the existence of compensatory mechanisms in Drosophila sensory neuron-to-somatosensory interneuron wiring (Valdes-Aleman et al., 2021). Specifically, genetically mispositioned sensory neurons form synapses with many of their normal interneuron partners, which grow abnormally to reach the mispositioned sensory neurons (Fig. $6 D$ ). In control and EL eve mutants, we characterized the position of sensory neurons by labeling vibration and proprioceptive sensory neurons with iav-GAL4 and 0165GAL4 driving membrane GFP but find no differences in sensory neuron axon position (Fig. 6G,H).

Next, we tested the idea that eve expression is required for normal EL somatosensory stimulus encoding. Drosophila larvae are optically clear so we used calcium signals in intact larvae to monitor EL activity. Specifically, a larva expressing GCaMP6m in ELs was placed on a bed of agarose with a coverslip on top (Fig. 7A). In this preparation, larvae do not crawl, but they move, which stimulates proprioceptors, and sound can be used to stimulate vibration-sensitive chordotonal sensory neurons (Wreden et al., 2017). In a single recording, we imaged baseline EL fluorescence, EL activity while the sound is played, and return to baseline. In control, there are small amplitude changes in fluorescence intensity in ELs during periods of self-movement and large amplitude changes in response to vibration (Fig. 6B,D). In EL eve mutants, there is only bleaching of the calcium signal (Fig. 7C,E).

We conclude that in the absence of eve expression, EL interneurons no longer normally encode somatosensory stimuli.

\section{Upon loss of eve expression, EL outputs are anatomically and functionally reconfigured}

EL interneurons are necessary for normal Drosophila larval behavior (Heckscher et al., 2015). Late-born ELs contribute to a circuit that regulates left-right symmetrical crawling, and early-born ELs contribute to a circuit that triggers fast escape rolling (Wreden et al., 2017). The observation that Eve(-) EL axons are less extended raised the possibility that EL output circuits could be reconfigured (Fig. 3).

The specific output partners of EL interneurons are not well characterized, and so we determined the location of Eve(-) EL output synapses. In control and EL eve mutants, we visualized presynaptic nerve termini, using expression of a V5 epitope tagged Brunchpilot (BRP-V5) protein (Wagh et al., 2006; Chen et al., 2014). In control, BRP signal is concentrated in large puncta in the central intermediate and medial region of the neuropile (Fig. 8 $8 A^{\prime}$ ). However, in EL eve mutants, BRP signal is found diffusely throughout the neuropile (Fig. 8B'). To quantify this, we used Fas2 expression to subdivide the neuropile into 

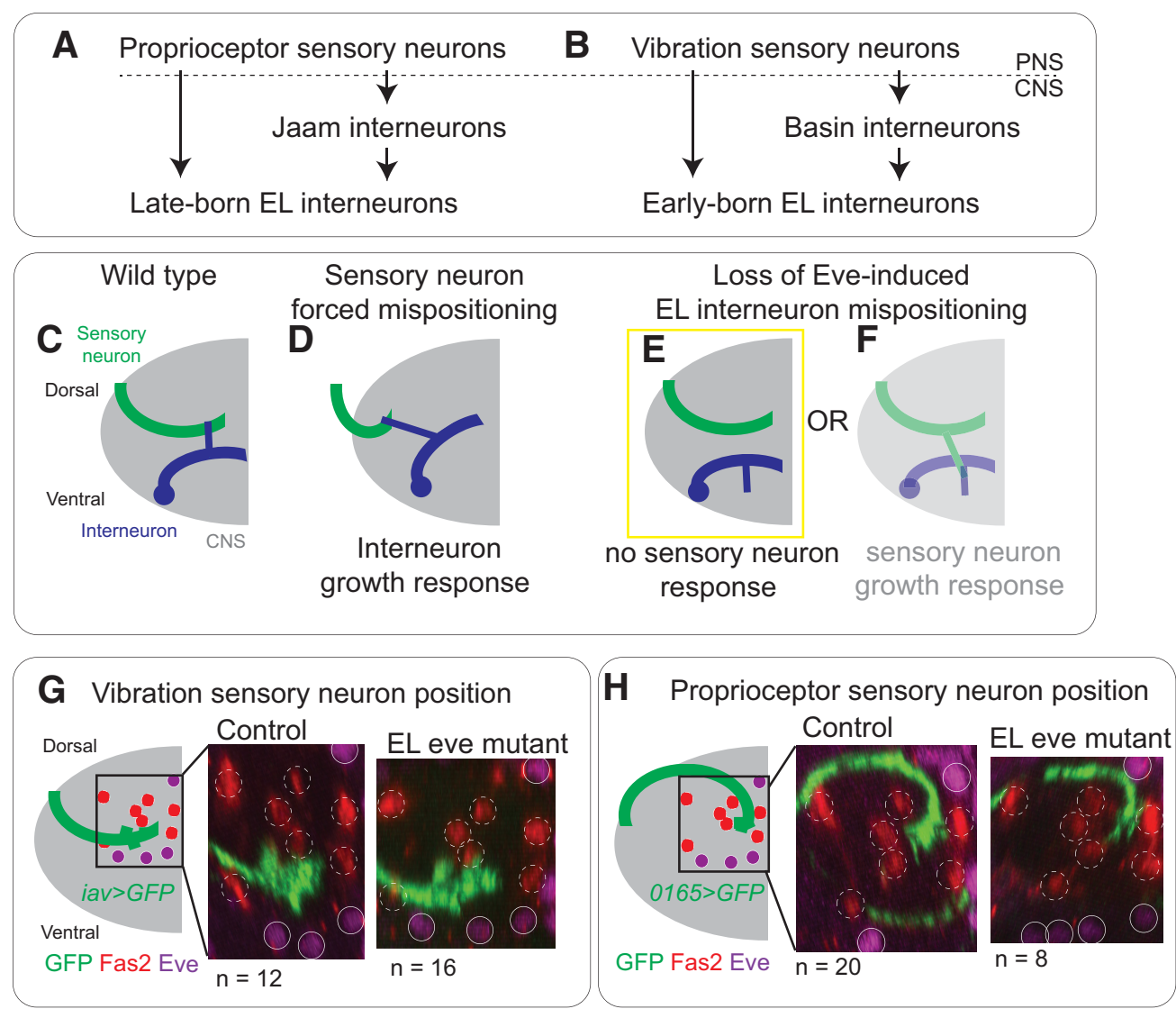

Figure 6. In EL eve mutants, there are no changes in sensory neurons axonal trajectories. $\boldsymbol{A}, \boldsymbol{B}$, Illustration of sensory inputs onto ELs. $\boldsymbol{A}$, Late-born ELs get direct input from proprioceptors and indirect input via the Jaam CNS interneurons. $\boldsymbol{B}$, Early-born ELs get direct input from mechanoreceptors (chordotonals) and indirect input via the Basin CNS interneurons. $\boldsymbol{C}-\boldsymbol{F}$, Illustrations of sensory neuron-to-interneuron wiring in different genetic conditions. $\boldsymbol{C}$, In wild-type, sensory neuron axons (green) and interneuron dendrites (blue) are in close enough proximity they can form synaptic contacts. $\boldsymbol{D}$, When sensory neuron axons are genetically mispositioned, interneurons grow in response, and the two cell types continue to form synaptic contacts. $\boldsymbol{E}, \boldsymbol{F}$, When EL interneuron dendrites are mispositioned because of lack of Eve, sensory neurons might or might not change position in response. $\boldsymbol{G}, \boldsymbol{H}$, Images of vibration and proprioceptive sensory neurons arbors. $\mathbf{G}, \boldsymbol{H}$, Axonal positions are similar in control and EL eve mutants for both vibration sensitive and proprioceptive sensory neurons. Single hemisegments of an L1 larval CNS are shown with dorsal up. Eve-expressing motor neurons are shown as solid circles with a diameter of five microns. Positions of Fas2 $(+)$ fascicles are shown as dashed circles. To the left of each image is a schematic of the axon position relative to landmarks. $n=$ the number of hemisegments scored. Genotypes: control in $\mathbf{G}$ is iav-GAL4/UAS-myr-GFP. EL eve mutant in $\mathbf{G}$ is $\triangle E L, D f(2 R) e v e / \triangle E L, D f(2 R) e v e ; i a v-G A L 4 / U A S-m y r-G F P$. Control in $\boldsymbol{H}$ is 0165-GAL4/UAS-myr-GFP. EL eve mutant in $\boldsymbol{H}$ is $\triangle E L, D f(2 R)$ eve/AEL, Df(2R)eve; 0165-GAL4/UAS-myr-GFP.

medial (M), intermediate (I), and lateral (L) zones, and scored for BRP signal in each zone (Fig. 8C,D). We find a significant increase in lateral signal in EL eve mutants compared with control. These data suggest that Eve(-) EL interneurons output synapses are relocated.

We used functional approaches to determine the extent to which relocation of Eve(-) EL output synapses change circuit function. We recorded spontaneously occurring crawling in control and in EL eve mutant larvae (Fig. 9). We calculated crawling speed as centroid position over time and left-right body symmetry using the angles between centroid and head position and centroid and tail position. EL eve mutants crawl significantly slower than control (Fig. 9A-C) with left-right asymmetrical body posture (Fig. 9D,F-H). We had previously recorded the behavior of larvae that entirely lack EL interneurons, and here we noticed that, in comparison, EL eve mutant larvae have more severe crawling defects (Heckscher et al., 2015). This is quantified as significantly greater left-right asymmetry in larvae with Eve(-) ELs than in larvae without ELs (Fig. 9D-H). The discrepancy between severity of the phenotypes could be explained by the idea that mispositioned $\operatorname{Eve}(-)$ EL output synapses make new, functional connections, which could provide a dominant negative effect at the circuit level (see Discussion).

The crawling defects provide strong functional evidence that eve expression is required in EL interneurons for normal somatosensory circuit function (Fig. 9). However, we demonstrated that in Eve(-) ELs sensory encoding is disrupted (Fig. 7). Therefore, it is possible that dysfunction in sensory encoding could explain the observed behavioral deficits. And so, we assayed the behavioral response to optogenetic stimulation of Eve(-) ELs . Optogenetics uses light to activate neurons and bypasses 

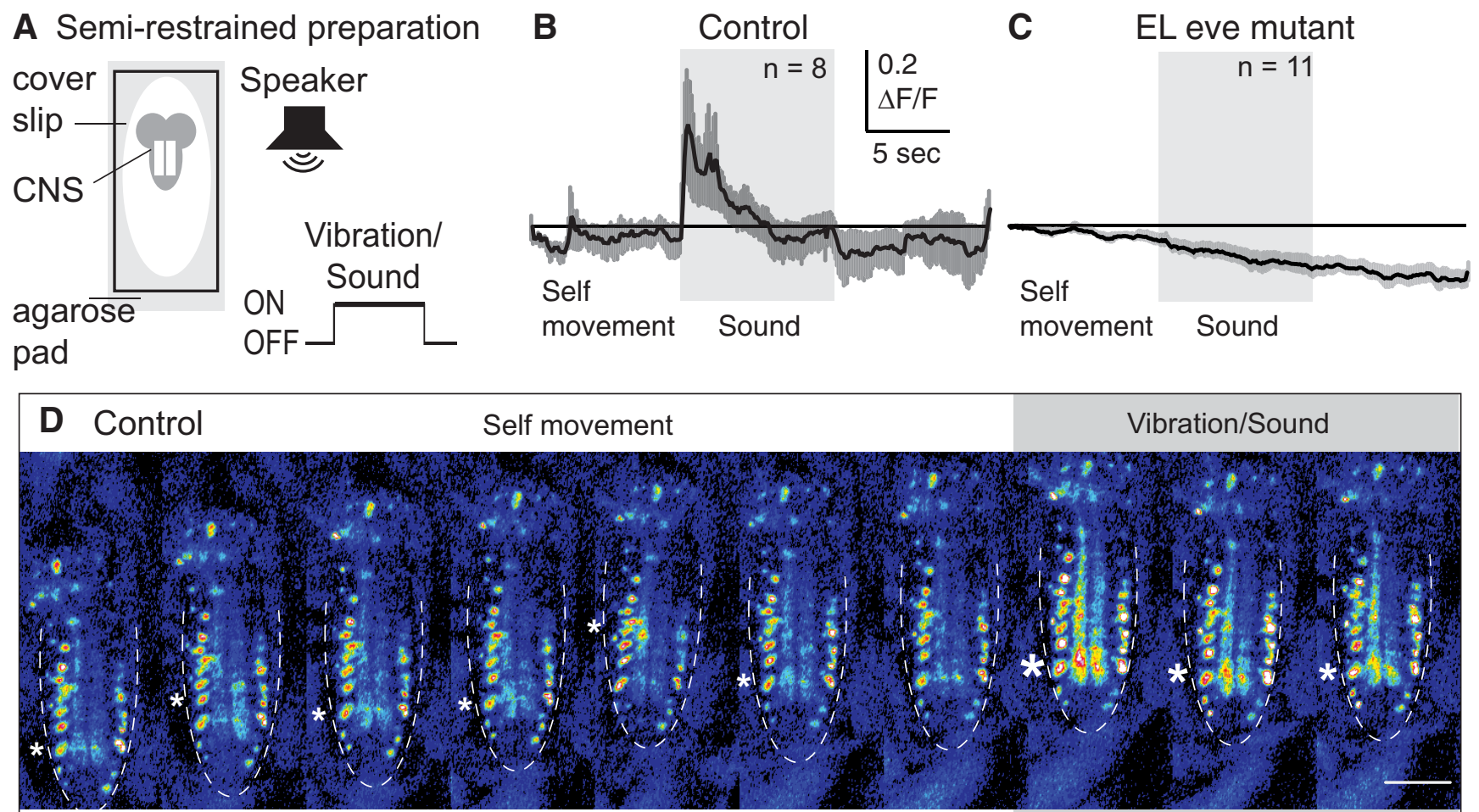

\section{E EL eve mutant}

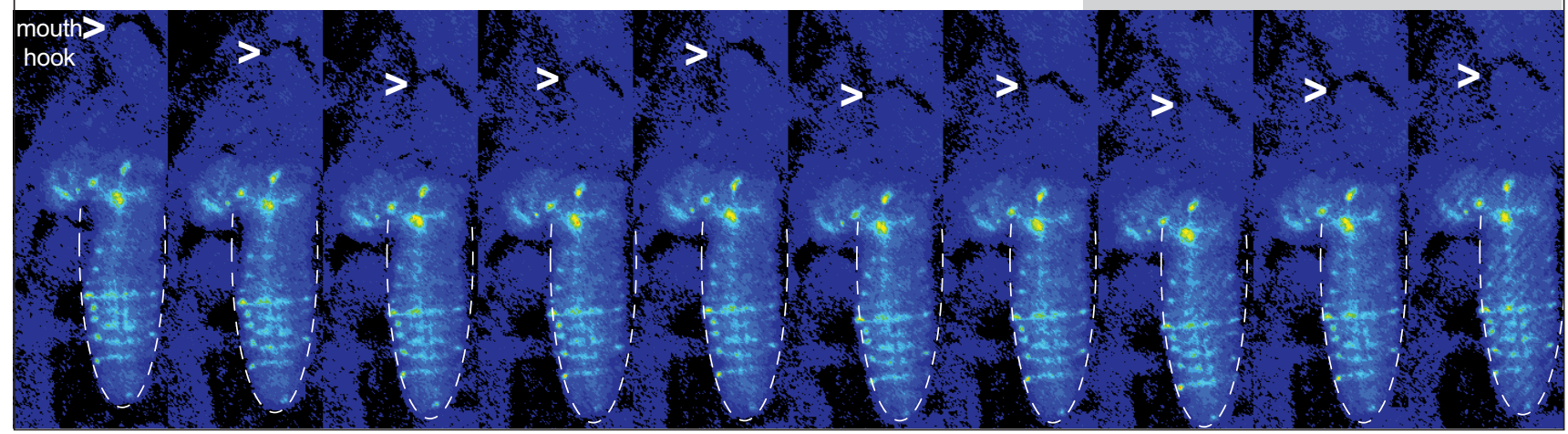

Figure 7. EL interneurons require eve expression to encode somatosensory stimuli. $\boldsymbol{A}$, Illustration of the semi-restrained preparation and stimulus protocol. Fluorescence in the CNS (gray lobed structure with two white lines representing neuropil) is recorded before, during, and after a sound is played from a speaker. B, C, Quantifications of EL calcium signals. B, In control, EL have small amplitude, dynamic calcium signals before sound onset, which corresponds to periods of self-movement. There are large amplitude changes in EL calcium signal on sound/vibration stimuli. $\Delta F / F$ is the change in fluorescence over baseline. $\boldsymbol{C}$, In EL eve mutants, ELs do not respond to stimuli. Averages (dark line) and SEM (light line) are shown. Scale for both is shown as an inset. $n=$ number of larvae recorded. $\boldsymbol{D}, \boldsymbol{E}$, Images from representative recordings of calcium signals in ELs. Fluorescence images are shown in pseudo-color with white/red as high fluorescence intensity and blue as low. Anterior is up with a scale bar of $100 \mu \mathrm{m}$. Dashed lines show the outline of the nerve cord. In $\boldsymbol{D}$, asterisk denotes region of nerve cord neuropile (central region) with increased fluorescence. In $\boldsymbol{E}$, $>$ points to mouth hooks. Genotypes: control is UAS-FLP, act5C-FRT.stop-GAL4; $\triangle E L, D f(2 R)$ eve/+; EL-GAL4/UAS-GCaMP6m. EL eve mutant is UAS-FLP, act5C-FRT.stop-GAL4; $\triangle E L, D f(2 R)$ eve/DEL, Df(2R)eve; EL-GAL4/UAS-GCaMP6m.

the need for any input from upstream neurons. We reasoned that optogenetic stimulation of Eve(-) ELs might elicit a novel behavior, consistent with the idea that Eve(-) EL output synapses are remapped. In control and EL eve mutants, we expressed the optogenetic effector, CsChrimson, and fed larvae all trans retinal (ATR), a cofactor needed for CsChrimson light sensitivity. As expected, in response to light, any larva not fed ATR fails to respond (Fig. 10D,E). Also, as expected, control larvae fed ATR roll on light exposure, which is quantified as fast centroid movement over time (Fig. 10A,D,F). In EL eve mutants fed ATR, in response to light, larvae preform a novel behavior-a dorsal body bend. This is quantified as a small increase in centroid movement over time (Fig. 10B,E,G). The body bend is an extreme $\mathrm{C}$ shape where the head and tail nearly touch, and to our knowledge, does not occur naturally in Drosophila melanogaster. Dorsal bending is robust, displayed by nearly 


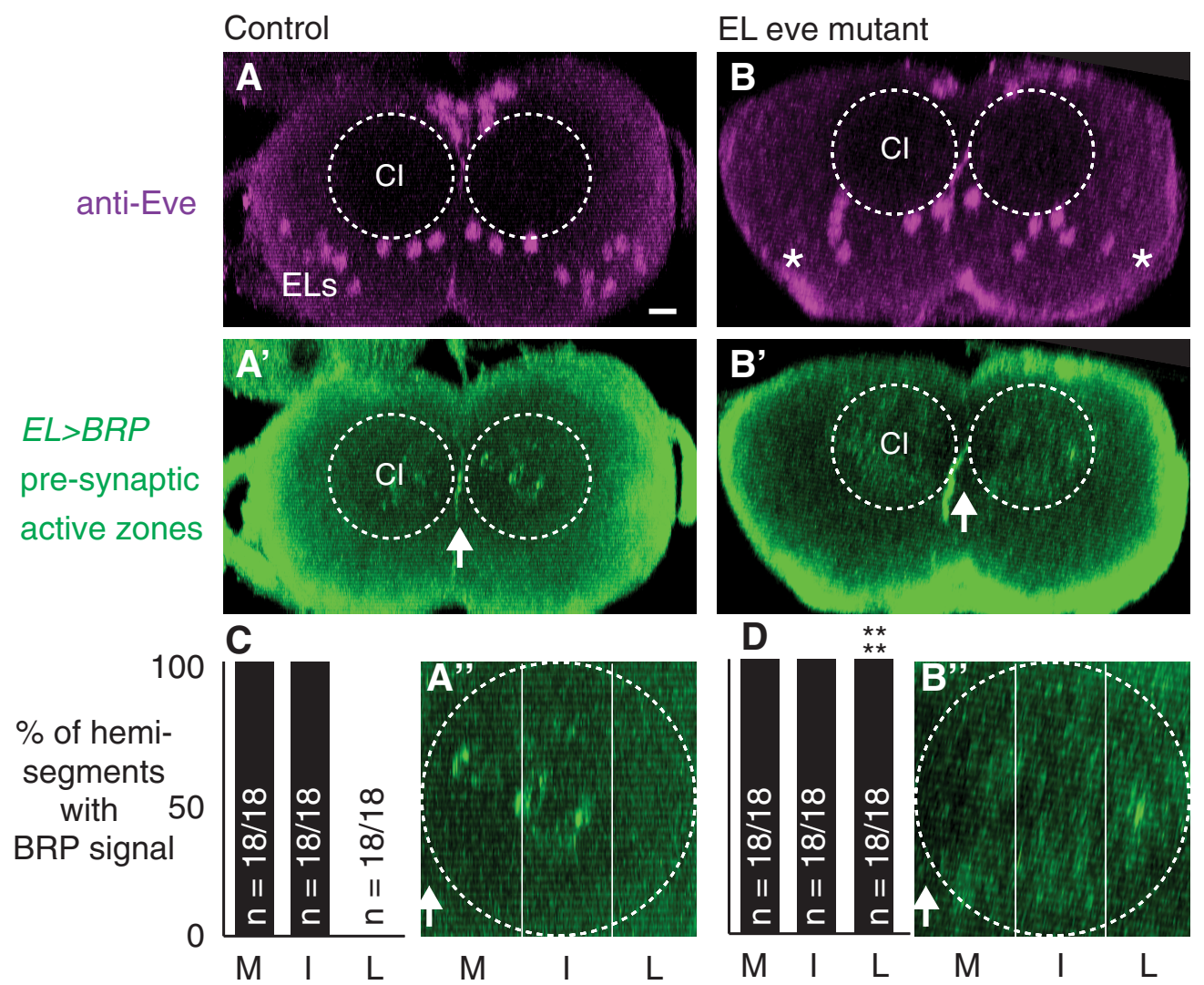

Figure 8. In Eve(-) ELs, output synapses are anatomically repositioned. $\boldsymbol{A}, \boldsymbol{B}$, Images of tagged presynaptic active zones. Eve labels

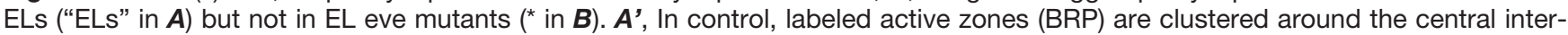
mediate Fas2 (+) fascicles $(\mathrm{Cl})$. $\boldsymbol{B}^{\prime}$, In EL eve mutants, BRP signal is diffuse throughout the entire neuropile. $\boldsymbol{A}-\boldsymbol{A}^{\prime \prime}$ are the same CNS and $\boldsymbol{B}-\boldsymbol{B}^{\prime \prime}$ are the same CNS. $\boldsymbol{A}^{\prime \prime}, \boldsymbol{B}^{\prime \prime}$ are a magnifications of the neuropile from one hemisegment in $\boldsymbol{A}^{\prime}$ or $\boldsymbol{B}^{\prime}$, respectively. Images are overlaid with lines showing medial (M), intermediate (I), and lateral (L) zones. Images show the CNS in cross section with dorsal up. Arrow denotes midline. Neuropile is outlined by a dashed circle. Scale bar: $10 \mu \mathrm{m}$. $\boldsymbol{C}, \boldsymbol{D}$, Quantifications of BRP signal distribution. $n=$ number of hemisegments with BRP signal above background within a given region/total number of hemisegments scored. Zones scored were medial (M), intermediate (I), and lateral (L) as shown in $\boldsymbol{A}^{\prime \prime}$, B'. Genotype: control is UAS-FLP, act5C-FRT.stopGAL4; $\triangle E L, D f(2 R)$ eve/+; EL-GAL4/UAS-FLP, BRP-frt-stop-frt-V5-2A-LexA. EL eve mutant is UAS-FLP, act5C-FRT.stop-GAL4; $\triangle E L, D f(2 R)$ eve/ $\triangle E L, D f(2 R)$ eve; EL-GAL4/UAS-FLP, BRP-frt-stop-frt-V5-2A-LexA.

every EL eve mutant larva. This demonstrates that Eve(-) EL output synapses are functional and that their activation leads to a novel behavior.

Activation of Eve(-) EL interneurons could lead to novel behavior for two reasons. First, EL output synapses could be remapped to new, downstream targets and away from roll inducing circuits. Alternatively, circuits mediating rolling and other sensorimotor transformations could be generally dysfunctional. This possibility needed to be tested because homeodomain proteins, including Eve, can have nonautonomous effects (Lee et al., 2019). To probe the function of rolling circuits, we provided a body wall pinch, which served as a mechanical and noxious stimulus, to both control and EL eve mutants (Hwang et al., 2007). In both genotypes, larvae roll in response to pinch (Fig. $10 H$ ). Thus, in EL eve mutant larvae, roll inducing circuits are not disrupted. Further, we found that both EL eve mutant and control larvae respond to vibration with a hunch (Fig. 10/). Thus, in EL eve mutant larvae, somatosensory stimuli can be perceived and transformed into appropriate motor output, and somatosensory circuits are not generally dysfunctional. We conclude that, in Eve(-) ELs, output synapses are no longer mapped to roll inducing circuits but instead to novel targets.

\section{Discussion}

In this study, we removed the conserved, homeodomain transcription factor, Eve, from Drosophila Eve-expressing ELs (Fig. 1). We found that eve regulates multiple aspects of EL interneuron morphogenesis (Figs. 2-4), and that eve is required for the proper integration of ELs into somatosensory circuits at both the input (Figs. 6, 7) and output (Figs. 8-10) levels.

\section{Previously undescribed roles for eve in neuronal morphogenesis}

Here, we show that eve expression is required for positioning EL interneuron neurites in all three axes (i.e., medial-lateral, anterior-posterior, and dorsal-ventral; Figs. 2-4). In Drosophila, each axis is patterned by a separate ligand/receptor signaling system (Zlatic et al., 2009; Evans and 


\section{Crawling speed}

A Control

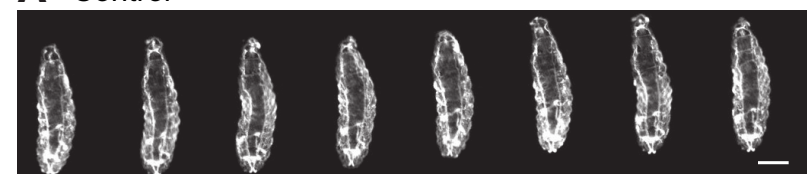

B EL eve mutant

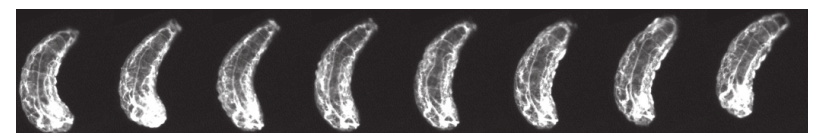

C

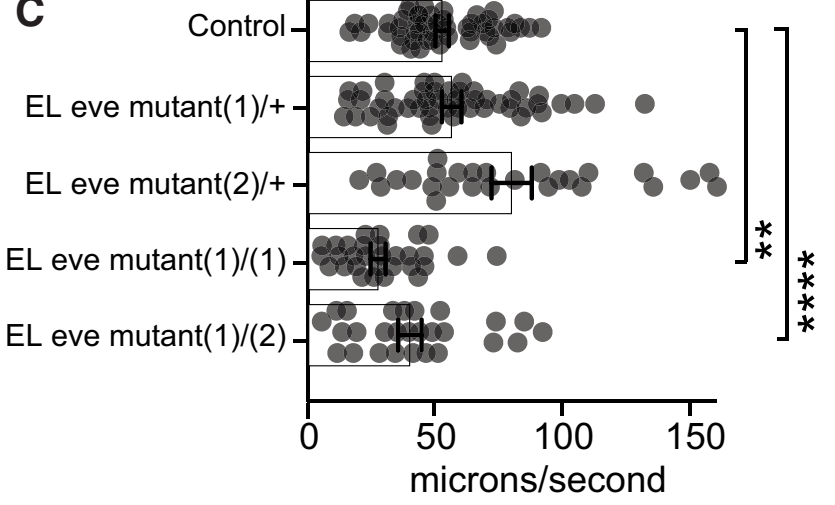

Left-right asymmetry
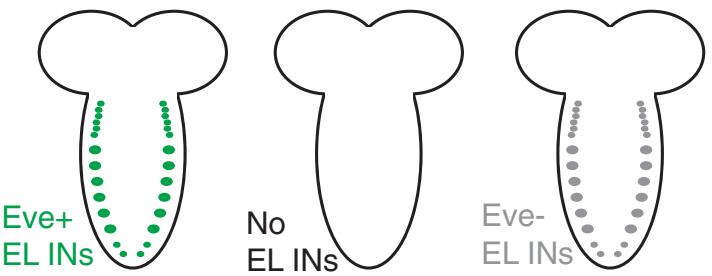

Control

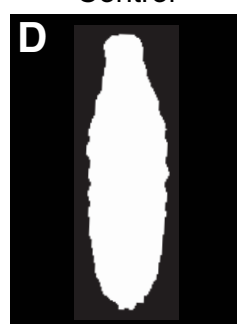

No ELs

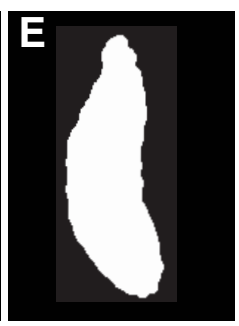

EL eve mutant

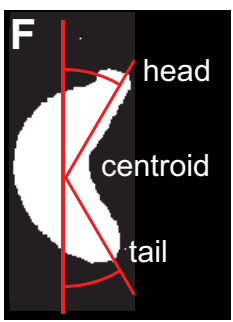

G Tail-to-centroid angle

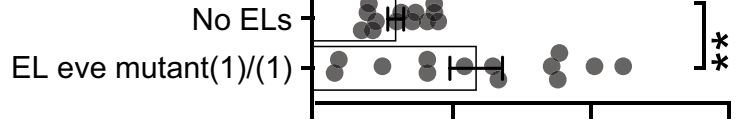

H Head-to-centroid angle

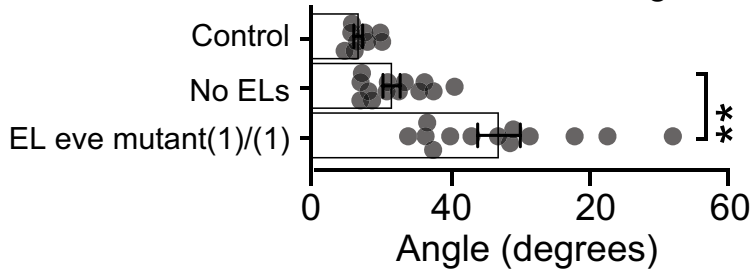

Figure 9. In EL eve mutants, there are defects in spontaneously-occurring crawling behavior. $\boldsymbol{A}-\boldsymbol{C}$, Images and quantification of larva crawling. $\boldsymbol{A}, \boldsymbol{B}$, Images of control and EL eve mutants during forward crawling. Images are frames $(0.66-\mathrm{s}$ intervals) from representative behavioral recordings. Anterior is up and scale bar is $150 \mu \mathrm{m}$. C. Quantification of crawling speed is calculated as centroid movement over time. Each dot represents the average data for one larva. Bars represent average of all data points, and whiskers show SEM. One-way ANOVA with Dunnett's multiple comparison; ${ }^{\star \star} p<0.01$, ${ }^{\star \star \star \star} p<0.0001$. $\boldsymbol{D}-\boldsymbol{F}$, Images of left-right asymmetrical body posture. $\boldsymbol{D}$, In control, larvae crawl left-right symmetrically. $\boldsymbol{E}$, When ELs are genetically ablated during embryogenesis, larvae crawl with left-right asymmetrical body posture. $\boldsymbol{F}$, In EL eve mutants, when Eve is removed from ELs, but the EL neurons remain, larvae crawl with a significantly severe left-right asymmetrical body posture. Images are single representative frames from behavioral recordings showing body shape with anterior up and scale bar of $40 \mu \mathrm{m}$. $\boldsymbol{F}$ is overlaid to show how angles are calculated. $\boldsymbol{G}, \boldsymbol{H}$, Quantification of left-right body asymmetry. Asymmetry is measured as tail-to-centroid and head-to-centroid angles during crawling. Each dot represents the average data for one larva. Data for No ELs replotted from Heckscher et al. (2015), with permission. Bars represent average of all data points, and whiskers show SEM ANOVA with Dunnett's multiple comparison test; ** $p<0.01$, Genotypes: Control is wildtype (+/+); no EL is UAS-RPR, UAS-HID/+,; EL-GAL4/+; refer to Figure 1D for naming of mutant allele genotypes used in this experiment.

Bashaw, 2010; Emerson et al., 2013). However, how individual interneurons read and interpret each signal is not well understood. Our data suggest eve is important for ELs to simultaneously read and/or interpret multiple ligand gradients simultaneously.

Generally, eve is considered a cell fate determinant. For example, in mouse V0v interneurons, evx1 represses expression of en1, a marker of $\mathrm{V} 1$ interneuron identities (Moran-Rivard et al., 2001). In VOv interneurons that lack evx1, en1 expression is derepressed and take on V1-like axonal projections. Similar fate changes are seen in Drosophila and C. elegans motor neurons when eve is disrupted (Landgraf et al., 1999; Esmaeili et al., 2002). Our data are more consistent with the idea that eve plays a role in the refinement of EL morphogenesis. In support for the morphogenetic refinement model is, first, in wild-type, there are no neurons with morphology that matches the morphology of Eve(-) ELs, as would be expected by a cell fate switching model. Second, there are no obvious largescale changes in gene expression, which are typically 

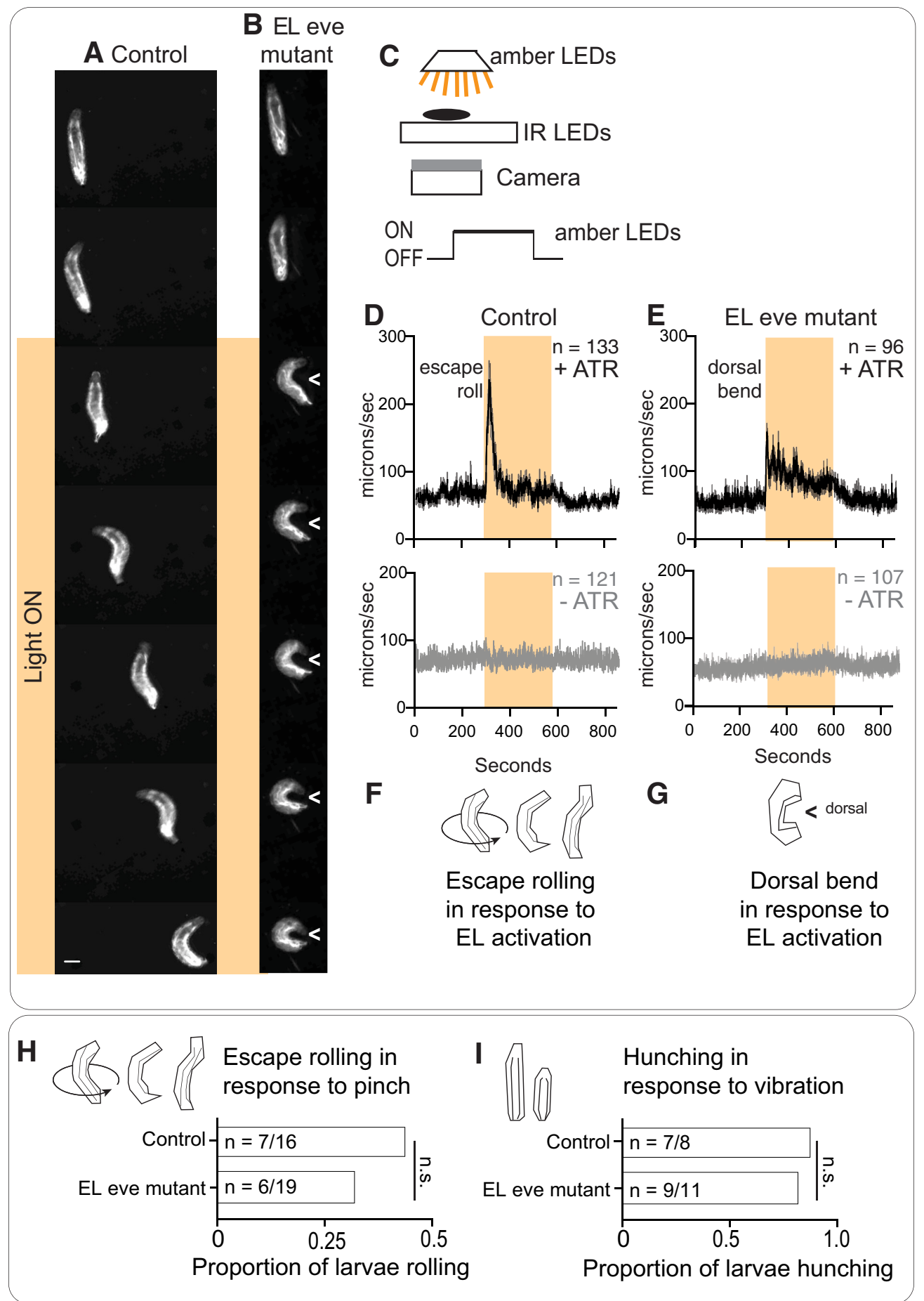

Figure 10. EL outputs are remapped in the absence of Eve. A, B, Images of behavioral responses to optogenetic stimulation of ELs. $\boldsymbol{A}$, Control larvae fed ATR and expressing CsChrimson in ELs roll in response to light. $\boldsymbol{B}$, EL eve mutant larvae fed ATR and expressing CsChrimson in ELs display a novel dorsal bend phenotype (can be either side). Images are frames from representative behavioral recordings (shown at 0.6-s intervals). Scale bar: $150 \mu \mathrm{m}$. In $\boldsymbol{B},>$ points to dorsal. $\boldsymbol{C}$, Illustration of the behavioral rig. The rig uses infrared light emitting diodes (IR LEDs) to illuminate larvae, which is detected by the camera, but not the larvae. Amber LEDs stimulate optogenetic effectors. D, E, Quantification of larval movement. Centroid speed is calculated as centroid displacement/ time. Orange bar shows exposure to amber light. Gray traces (bottom) are control larvae not fed ATR, a co-factor needed for optogenetic simulation. Black traces are experimental larvae, which were fed ATR. $n=$ number of larvae recorded. Average is shown as darker lines and SEM is shown as lighter lines. $\boldsymbol{F}, \mathbf{G}$, Illustrations of behavioral responses. Controls roll in response to EL activation, whereas EL eve mutants perform a dorsal bend in response to EL activation. $\boldsymbol{H}, \boldsymbol{I}$, Quantification of larval sensorimotor transformations. Control and EL eve mutant larvae roll in response to body wall pinch and hunch in response to vibration. Illustration of each behavior is shown in top left of each panel. For quantifications, $n=$ number of larvae responding to each stimulus over the total 
continued

number of larvae stimulated. Chi-squared test, n.s. = not significant. Genotype: control in $\boldsymbol{A}, \boldsymbol{D}$ is UAS-FLP, act5C-FRT.stop-GAL4; $\triangle E L, D f(2 R) e v e /+;$ EL-GAL4/UAS-Cs.Chrimson.mVenus. EL eve mutant in B and E is UAS-FLP, act5C-FRT.stop-GAL4; $\triangle E L, D f(2 R)$ eve/AEL, Df(2R)eve; EL-GAL4/UAS-Cs.Chrimson.mVenus; control in $\boldsymbol{H}$ is $\Delta E L, D f(2 R) e v e /+$. EL eve mutant in I is $\Delta E L, D f(2 R) e v e) /$ $\triangle E L, D f(2 R)$ eve.

associated with cell fate changes (Fig. 5). Third, eve expression in ELs is not playing a role in initial morphogenesis (see next paragraph for more).

Both Eve(-) and Eve(+) ELs cross the midline at embryonic stage 15 (Fig. $2 A-C$ ). Thus, eve expression is either dispensable for initial morphogenesis, or in EL eve mutants there is an undetectable pulse of early eve expression in ELs. But, we and others have not found Eve protein expression in ELs in EL eve mutants at any stage of development (Fig. 1A,B; Fujioka et al., 2003). In later stage embryos and larvae, we observe morphologic defects in Eve(-) ELs (Figs. 2D-H, 3, 4). This raises the possibility that, in general, eve genes may play a later role in morphogenesis. This is consistent with the observation that, in mouse V0v interneurons, there is early evx1 expression and later evx2 expression. However, the later role of evx2 is unknown (Moran-Rivard et al., 2001).

In general, eve genes are known to regulate axon morphogenesis (Doe et al., 1988; Landgraf et al., 1999; Moran-Rivard et al., 2001; Esmaeili et al., 2002; Fujioka et al., 2003). In this study, we show that late-born Eve(-) ELs have axonal defects (Figs. 3, 4). Notably, the role of eve in dendrite morphogenesis is extremely poorly characterized. The distinction between dendrite and axon is important because these two compartments carry out different functions. Further, in Drosophila, interneuron axons and dendrites are structurally different. Dendrites are often highly branched, and lack mitochondria and postsynaptic machinery. Whereas, axon terminals (boutons) are full of mitochondria, pools of synaptic vesicles, microtubules, and vesicle release sites. Each part of the arbor (axon or dendrite) can be independently controlled by different transcription factors (Kurmangaliyev et al., 2019). For example, in Drosophila sensory neurons, the transcription factors Knot and Cut specifically regulate dendrite morphogenesis, but not axonal morphology (Jinushi-Nakao et al., 2007). Thus, in Drosophila, axon and dendrite morphology can be controlled as independent modules. Here, we show that in addition to regulating axon morphology, eve regulates dorsal-ventral dendrite positioning (Fig. 4). eve expression is also required for dendrite morphogenesis in RP motor neurons (Fujioka et al., 2003). Taken together, our data show that eve coordinately regulates multiple aspects of neuronal morphogenesis, and that coordinate control may be a widely-occurring role for neuronal eve expression.

\section{eve expression in ELs plays a role in somatosensory circuit assembly}

Neuronal circuits are functional units of the nervous system. Sensorimotor circuits, specifically, transform somatosensory stimuli into motor output. Therefore, functional assays are required for the study of somatosensory circuit assembly. However, because the circuit context of individual interneurons is not well characterized, often researchers rely on anatomic assays to infer changes at the circuit level. One reason an anatomic approach can be flawed is the existence of compensatory mechanisms that allow for relatively normal circuit wiring despite changes in neuron morphology (Landgraf et al., 1999; Meng and Heckscher, 2021; Valdes-Aleman et al., 2021). In this study, we link defects in neuronal morphology to changes in circuit function, thereby explicitly demonstrating the role of eve expression in somatosensory circuit assembly.

\section{The role of eve expression in formation of functional input synapses}

We show that eve is required for somatosensory stimulus encoding by ELs (Fig. 7). Based on known connectivity of ELs with other neurons (Fig. 6A), we infer that in ELs, eve is required for the formation of at least four types of functional input synapses: those from vibration (chordotonal) sensory neurons to early-born ELs, from vibrationsensitive interneurons (Basins) to early-born ELs, from proprioceptive sensory neurons to late-born ELs, and from proprioceptive-sensitive interneurons (Jaams) to late-born ELs. The likely cell biological underpinning, at least for late-born ELs, is that axons from input sensory neurons (Fig. 6G,H) are not in close enough proximity to make synaptic contact with Eve(-) ELs (Fig. 4). Because of technical limitations, we could not visualize dendrite morphology of early-born ELs.

In the Drosophila nerve cord, there is unidirectional compensatory growth from interneurons to genetically misplaced sensory neurons (Valdes-Aleman et al., 2021; Fig. 6C,D). Thus, Drosophila sensory neuron-to-interneuron wiring can be robust to morphologic alterations to circuit components. The observation (Fig. 6G,H) that sensory neurons do not grow to reach mispositioned Eve(-) EL dendrites raises two possibilities: (1) in this system, compensatory growth is unidirectional (i.e., interneurons grow to misplaced sensory neurons, but not vice versa); and (2) alternatively, compensatory growth is bidirectional, however, eve expression is required for this process. Future experiments will be needed to distinguish between these models.

\section{The role of eve in positioning output synapses}

Our data show Eve(-) EL output synapses are functional, but remapped. Spontaneously-occurring crawling behavior is disrupted in EL eve mutants (Fig. 7), and that this disruption is significantly worse than in larvae which lack EL neurons altogether. This could be explained by requirement for ELs during early circuit development (e.g., acting as a scaffold for normal axonal pathfinding for other neurons). Alternatively, mature Eve(-) ELs could 
exert a dominant negative effect at the level of circuit function. We favor the latter idea because it is consistent with the optogenetic experiments presented in Figure 9, and the anatomic data presented in Figure 7. Figure 7 shows that in controls, EL output synapses are excluded from many zones of the neuropile including the dorsal lateral zone, which houses the dendrites of dorsally-projecting motor neurons. However, Eve(-) ELs are likely to form output synapses in this region. This specific re-distribution of output synapses is notable because it raises the possibility that Eve(-) ELs output synapses (ELs are excitatory) could be directly re-mapped to dorsal motor neurons. Such a re-mapping could explain the novel behavioral phenotype, dorsal body bending phenotype seen on optogenetic activation of Eve(-) ELs in Figure 9. Regardless of the exact anatomic changes, the data in Figure 9 show that output synapses of $\operatorname{Eve}(-)$ ELs are functional, but are functionally re-mapped to new output circuits.

In conclusion, we have provided an updated understanding of the role of eve expression in neurons. Our data provide understanding of the role of neuronal eve at the levels of circuit physiology and animal behavior. Further they provide insight into the genetic logic of somatosensory circuit assembly, demonstrating that multiple terminal neuronal features can be coordinately regulated by the activity of a single postmitotic transcription factor. Finally, our data raises new questions about the role of eve expression in other neuron types and enable future experimental inquiry into somatosensory circuit assembly in Drosophila.

\section{References}

Broihier HT, Skeath JB (2002) Drosophila homeodomain protein $\mathrm{dHb} 9$ directs neuronal fate via crossrepressive and cell-nonautonomous mechanisms. Neuron 35:39-50.

Chen Y, Akin O, Nern A, Tsui CY, Pecot MY, Zipursky SL (2014) Celltype-specific labeling of synapses in vivo through synaptic tagging with recombination. Neuron 81:280-293.

Clark MQ, Zarin AA, Carreira-Rosario A, Doe CQ (2018) Neural circuits driving larval locomotion in Drosophila. Neural Dev 13:6.

Couton L, Mauss AS, Yunusov T, Diegelmann S, Evers JF, Landgraf M (2015) Development of connectivity in a motoneuronal network in Drosophila larvae. Curr Biol 25:568-576.

D'Elia KP, Dasen JS (2018) Development, functional organization, and evolution of vertebrate axial motor circuits. Neural Dev 13:10.

Doe CQ, Smouse D, Goodman CS (1988) Control of neuronal fate by the Drosophila segmentation gene even-skipped. Nature 333:376378.

Emerson MM, Long JB, Van Vactor D (2013) Drosophila semaphorin2 $b$ is required for the axon guidance of a subset of embryonic neurons. Dev Dyn 242:861-873.

Esmaeili B, Ross JM, Neades C, Miller DM 3rd, Ahringer J (2002) The C. elegans even-skipped homologue, vab-7, specifies DB motoneurone identity and axon trajectory. Development 129:853-862.

Evans TA, Bashaw GJ (2010) Axon guidance at the midline: of mice and flies. Curr Opin Neurobiol 20:79-85.

Frasch M, Hoey T, Rushlow C, Doyle H, Levine M (1987) Characterization and localization of the even-skipped protein of Drosophila. EMBO J 6:749-759.

Ferrier DE, Minguillón C, Cebrián C, Garcia-Fernàndez J (2001) Amphioxus Evx genes: implications for the evolution of the midbrain-hindbrain boundary and the chordate tailbud. Dev Biol 237:270-281.

Fujioka M, Emi-Sarker Y, Yusibova GL, Goto T, Jaynes JB (1999) Analysis of an even-skipped rescue transgene reveals both composite and discrete neuronal and early blastoderm enhancers, and multi-stripe positioning by gap gene repressor gradients. Development 126:2527-2538.

Fujioka M, Lear BC, Landgraf M, Yusibova GL, Zhou J, Riley KM, Patel NH, Jaynes JB (2003) Even-skipped, acting as a repressor, regulates axonal projections in Drosophila. Development 130: 5385-5400.

Hattori Y, Sugimura K, Uemura T (2007) Selective expression of Knot/Collier, a transcriptional regulator of the EBF/OIf-1 family, endows the Drosophila sensory system with neuronal class-specific elaborated dendritic patterns. Genes Cells 12:1011-1022.

Heckscher ES, Long F, Layden MJ, Chuang CH, Manning L, Richart J, Pearson JC, Crews ST, Peng H, Myers E, Doe CQ (2014) Atlasbuilder software and the eNeuro atlas: resources for developmental biology and neuroscience. Development 141:2524-2532.

Heckscher ES, Zarin AA, Faumont S, Clark MQ, Manning L, Fushiki A, Schneider-Mizell CM, Fetter RD, Truman JW, Zwart MF, Landgraf M, Cardona A, Lockery SR, Doe CQ (2015) EvenSkipped $(+)$ interneurons are core components of a sensorimotor circuit that maintains left-right symmetric muscle contraction amplitude. Neuron 88:314-329.

Hwang RY, Zhong L, Xu Y, Johnson T, Zhang F, Deisseroth K, Tracey WD (2007) Nociceptive neurons protect Drosophila larvae from parasitoid wasps. Curr Biol 17:2105-2116.

Jinushi-Nakao S, Arvind R, Amikura R, Kinameri E, Liu AW, Moore AW (2007) Knot/Collier and cut control different aspects of dendrite cytoskeleton and synergize to define final arbor shape. Neuron 56:963-978.

Juárez-Morales JL, Schulte CJ, Pezoa SA, Vallejo GK, Hilinski WC, England SJ, de Jager S, Lewis KE (2016) Evx1 and Evx2 specify excitatory neurotransmitter fates and suppress inhibitory fates through a Pax2-independent mechanism. Neural Dev 11:5.

Kohsaka H, Guertin PA, Nose A (2017) Neural circuits underlying fly larval locomotion. Curr Pharm Des 23:1722-1733.

Kurmangaliyev YZ, Yoo J, LoCascio SA, Zipursky SL (2019) Modular transcriptional programs separately define axon and dendrite connectivity. eLife 8:e50822.

Lai HC, Seal RP, Johnson JE (2016) Making sense out of spinal cord somatosensory development. Development 143:3434-3448.

Landgraf M, Roy S, Prokop A, VijayRaghavan K, Bate M (1999) evenskipped determines the dorsal growth of motor axons in Drosophila. Neuron 22:43-52.

Landgraf M, Sánchez-Soriano N, Technau GM, Urban J, Prokop A (2003) Charting the Drosophila neuropile: a strategy for the standardised characterisation of genetically amenable neurites. Dev Biol 260:207-225.

Lee EJ, Kim N, Park JW, Kang KH, Kim WI, Sim NS, Jeong CS, Blackshaw S, Vidal M, Huh SO, Kim D, Lee JH, Kim JW (2019) Global analysis of intercellular homeodomain protein transfer. Cell Rep 28:712-722.e3.

Meng JL, Heckscher ES (2021) Development of motor circuits: from neuronal stem cells and neuronal diversity to motor circuit assembly. Curr Top Dev Biol 142:409-442.

Meng JL, Marshall ZD, Lobb-Rabe M, Heckscher ES (2019) How prolonged expression of Hunchback, a temporal transcription factor, re-wires locomotor circuits. eLife 8:e46089.

Meng JL, Wang Y, Carrillo RA, Heckscher ES (2020) Temporal transcription factors determine circuit membership by permanently altering motor neuron-to-muscle synaptic partnerships. eLife 9: e56898.

Moran-Rivard L, Kagawa T, Saueressig H, Gross MK, Burrill J, Goulding M (2001) Evx1 is a postmitotic determinant of v0 interneuron identity in the spinal cord. Neuron 29:385-399.

Nern A, Pfeiffer BD, Rubin GM (2015) Optimized tools for multicolor stochastic labeling reveal diverse stereotyped cell arrangements in the fly visual system. Proc Natl Acad Sci USA 112:E2967-E2976. 
Ohyama T, Schneider-Mizell CM, Fetter RD, Aleman JV, Franconville R, Rivera-Alba M, Mensh BD, Branson KM, Simpson JH, Truman JW, Cardona A, Zlatic M (2015) A multilevel multimodal circuit enhances action selection in Drosophila. Nature 520:633-639.

Parrish JZ, Kim MD, Jan LY, Jan YN (2006) Genome-wide analyses identify transcription factors required for proper morphogenesis of Drosophila sensory neuron dendrites. Genes Dev 20:820-835.

Pym EC, Southall TD, Mee CJ, Brand AH, Baines RA (2006) The homeobox transcription factor Even-skipped regulates acquisition of electrical properties in Drosophila neurons. Neural Dev 1:3.

Rexed B (1952) The cytoarchitectonic organization of the spinal cord in the cat. J Comp Neurol 96:415-495.

Risse B, Berh D, Otto N, Klämbt C, Jiang X (2017) FIMTrack: an open source tracking and locomotion analysis software for small animals. PLoS Comput Biol 13:e1005530.

Santiago C, Bashaw GJ (2014) Transcription factors and effectors that regulate neuronal morphology. Development 141:4667-4680.

Sholl DA (1953) Dendritic organization in the neurons of the visual and motor cortices of the cat. J Anat 87:387-406.

Stratmann J, Ekman H, Thor S (2019). A branching gene regulatory network dictating different aspects of a neuronal cell identity. PLoS Genet 13:e1006729.

Valdes-Aleman J, Fetter RD, Sales EC, Heckman EL, Venkatasubramanian L, Doe $C Q$, Landgraf $M$, Cardona $A$, Zlatic M (2021) Comparative connectomics reveals how partner identity, location, and activity specify synaptic connectivity in Drosophila. Neuron 109:105-122.e7.

Wagh DA, Rasse TM, Asan E, Hofbauer A, Schwenkert I, Dürrbeck H, Buchner S, Dabauvalle M-C, Schmidt M, Qin G, Wichmann C, Kittel R, Sigrist SJ, Buchner E (2006) Bruchpilot, a protein with homology to ELKS/CAST, is required for structural integrity and function of synaptic active zones in Drosophila. Neuron 49:833844.

Wreden CC, Meng JL, Feng W, Chi W, Marshall ZD, Heckscher ES (2017) Temporal cohorts of lineage-related neurons perform analogous functions in distinct sensorimotor circuits. Curr Biol 27:15211528.e4.

Zarin AA, Asadzadeh J, Hokamp K, McCartney D, Yang L, Bashaw GJ, Labrador JP (2014) A transcription factor network coordinates attraction, repulsion, and adhesion combinatorially to control motor axon pathway selection. Neuron 81:1297-1311.

Zeilig G, Enosh S, Rubin-Asher D, Lehr B, Defrin R (2012) The nature and course of sensory changes following spinal cord injury: predictive properties and implications on the mechanism of central pain. Brain 135:418-430. doi:10.1093/brain/awr270

Zlatic M, Landgraf M, Bate M (2003) Genetic specification of axonal arbors: atonal regulates robo3 to position terminal branches in the Drosophila nervous system. Neuron 37:41-51.

Zlatic M, Li F, Strigini M, Grueber W, Bate M (2009) Positional cues in the Drosophila nerve cord: semaphorins pattern the dorso-ventral axis. PLoS Biol 7:e1000135. 